\title{
Application of a Beamforming Technique to the Measurement of Airfoil Leading Edge Noise
}

\author{
Thomas Geyer, Ennes Sarradj, and Jens Giesler \\ Aeroacoustics Group, Brandenburg University of Technology, 03046 Cottbus, Germany \\ Correspondence should be addressed to Thomas Geyer, thomas.geyer@tu-cottbus.de \\ Received 15 July 2011; Accepted 26 October 2011 \\ Academic Editor: Akira Ikuta
}

Copyright () 2012 Thomas Geyer et al. This is an open access article distributed under the Creative Commons Attribution License, which permits unrestricted use, distribution, and reproduction in any medium, provided the original work is properly cited.

The present paper describes the use of microphone array technology and beamforming algorithms for the measurement and analysis of noise generated by the interaction of a turbulent flow with the leading edge of an airfoil. Experiments were performed using a setup in an aeroacoustic wind tunnel, where the turbulent inflow is provided by different grids. In order to exactly localize the aeroacoustic noise sources and, moreover, to separate airfoil leading edge noise from grid-generated noise, the selected deconvolution beamforming algorithm is extended to be used on a fully three-dimensional source region. The result of this extended beamforming are three-dimensional mappings of noise source locations. Besides acoustic measurements, the investigation of airfoil leading edge noise requires the measurement of parameters describing the incident turbulence, such as the intensity and a characteristic length scale or time scale. The method used for the determination of these parameters in the present study is explained in detail. To demonstrate the applicability of the extended beamforming algorithm and the experimental setup as a whole, the noise generated at the leading edge of airfoils made of porous materials was measured and compared to that generated at the leading edge of a common nonporous airfoil.

\section{Introduction}

When an airfoil is subject to a turbulent flow, noise is basically generated at both the airfoil leading edge and the trailing edge. If the turbulence intensity in the inflow is higher than that of the turbulence generated within the boundary layer (which subsequently interacts with the trailing edge and generates trailing edge noise), the radiated aeroacoustic noise is dominated by sound generated at the leading edge of the airfoil. As early experiments on a flat plate showed, the existence of turbulence in the incoming flow can increase the noise radiation significantly [1]. Additionally, the frequency spectrum may be different for both noise source locations. For example, it was found that the noise from wind turbines is dominated at low frequencies by broadband inflow turbulence noise, while at higher frequencies noise due to the interaction of the turbulent boundary layer with the trailing edge of the blades becomes more important [2].

Airfoil leading edge noise, also referred to as incident turbulence interaction noise, is a result of turbulent structures which generate fluctuating forces that act on the airfoil.
Consequently, the investigation of airfoil leading edge noise not only requires acoustic measurements, but also requires some knowledge about the characteristics of the turbulent flow. This section gives a short overview on basic leading edge noise studies, thereby focusing on the experimental techniques used for the measurement of the generated noise and for the measurement of the turbulence characteristics.

In 1964, Sharland [1] examined both the broadband and the discrete frequency noise generation in axial flow fans and developed a theoretical model for the noise generated by a flat plate in a turbulent flow. He found good agreement between the predictions and noise measurements on an isolated $0.0254 \mathrm{~m}$ chord flat plate, which was positioned in the turbulent mixing region of an open jet shear layer. The acoustic measurements were performed using a single microphone at an angle of $90^{\circ}$ to the plate. Additional measurements were done for the noise generated by a fan rotor using a number of microphones in the acoustic far field, including the measurement of the mean axial velocity and turbulent velocity with a single-wire hot-wire probe upstream of the rotor. No length scale of the turbulent inflow was measured. 
An experimental study on the noise radiated by an airfoil due to incident turbulence was done by Fink $[3,4]$. The measurements were performed in grid-generated turbulence with an intensity of $4 \%$ and $6 \%$, respectively. Far field sound pressure levels were measured using 1/4 inch condenser microphones, located at three positions on an arc with a radius of $2.14 \mathrm{~m}$ in a vertical plane parallel to the flow, with the microphones at $60^{\circ}, 90^{\circ}$, and $120^{\circ}$ angular position relative to the direction of the flow. Additionally, surface pressure levels were measured. Streamwise and transverse turbulence intensity of the incident turbulence were found to be approximately equal. The streamwise integral length scale was obtained from the autocorrelation of the data from a single hot-wire probe, and spanwise integral length scale was derived from cross-correlation of hot-wire measurements with two probes.

A fundamental work on the noise generated by an airfoil subject to a turbulent flow was performed by Amiet [5], who states that, basically, the resulting noise is directly related to the unsteady pressure fluctuations on the airfoil surface. Amiet developed a noise prediction model by taking into account the cross-power spectral density of the surface pressure on the airfoil due to the inflow turbulence characterized by its energy spectrum. The part of the resulting model which applies to the high frequency range allows for the calculation of the third-octave band far field sound pressure level $\left(L_{p}\right)$ based on the airfoil semispan $h$, the Mach number $M a=$ $U / c$, the integral length scale of the turbulence $\Lambda$, and the (normalized) intensity of the turbulence $\sqrt{\overline{u^{2}}} / U$ :

$$
\begin{aligned}
L_{p}= & 10 \cdot \log _{10}\left(\frac{\Lambda \cdot h}{R^{2}} \cdot\left(\frac{U}{c}\right)^{5} \cdot \frac{\overline{u^{2}}}{U^{2}} \cdot \frac{\hat{K}_{x}^{3}}{\left(1+\hat{K}_{x}^{2}\right)^{7 / 3}}\right) \\
& +181.3 \mathrm{~dB} .
\end{aligned}
$$

In (1), $K_{x}$ is the chordwise turbulence wavenumber (which is normalized by the wavenumber range of the energy containing eddies, denoted by the $\Lambda$ symbol) and $R$ is the observer distance normal to the airfoil. This notation of Amiet's model is based on the assumption that the turbulence spectrum can be modeled by the Karman model for isotropic turbulence. The Amiet model includes a dependence on the fifth power of the Mach number and on the square of the turbulence intensity. The predicted third-octave band sound-pressure levels show reasonable agreement with data measured on a flat plate in an anechoic wind tunnel [4].

Paterson and Amiet [6] performed acoustic measurements on a NACA 0012 airfoil in an open jet wind tunnel. A square mesh grid generated nearly isotropic incident turbulence with a turbulence intensity in the order of $4 \%$ to $5 \%$, and hence the leading edge was identified as the dominant noise source region, resulting in the generation of broadband noise. The parameters of the turbulent inflow were obtained using hot-wire anemometry, with the airfoil removed, at a position corresponding to the airfoil midchord, which was considered to also apply to the leading edge position. The lateral and longitudinal integral length scales were determined from the autocorrelation of a single hotwire probe. Both scales were found to be nearly independent of the flow speed, with a ratio of lateral to longitudinal scale in the order of 0.8 , revealing an approximately homogeneous and nearly anisotropic turbulence field. The acoustic measurements were performed using condenser microphones located in the acoustic far field and a narrow bandwidth, real-time spectrum analyzer. Good agreement between the measured leading edge noise in comparison to the noise predicted by the model of Amiet [5] for low frequencies and high Mach numbers was observed.

The noise due to turbulent inflow was also discussed by Lowson [7] as a part of his wind turbine noise prediction model. The calculations are based on the model by Amiet [5], but Lowson proposes a simple formula for the transition between the high frequency part and the low frequency part of the leading edge noise spectrum considered by Amiet. The turbulence parameters were not measured, but predicted using simple models for the atmospheric boundary layer. The final predictions of the resulting wind turbine noise model, which includes other noise source mechanisms as well, were compared to single microphone measurements on two wind turbines.

A detailed study on the noise generation at both the leading edge and the trailing edge of six airfoils in an open jet wind tunnel was performed by Oerlemans and Migliore $[8,9]$, who used a planar 48 channel microphone array for the acoustic measurements. The required inflow turbulence for the generation of leading edge noise was provided by a grid, the turbulence intensity was measured in the empty test section at positions corresponding to the leading edge and trailing edge of the airfoil, respectively, using a crosswire hot-wire probe. The resulting leading edge noise was found to be independent of airfoil tripping, to scale with the sixth power of the flow speed and to increase with increasing "sharpness of the model leading edge."

The influence of the angle of attack on the leading edge noise generation of a flat plate and two airfoils was experimentally examined by Moreau and Roger [10]. Acoustic measurements were conducted with single microphones, located at several polar angles between $-105^{\circ}$ and $105^{\circ}$ with respect to the nozzle axis in the midspan plane of the airfoil. The turbulence with an intensity of $5 \%$ was generated by a grid made of struts of $0.01 \mathrm{~m}$ diameter. The streamwise velocity fluctuations were measured with a single-wire hot-wire probe with the airfoil removed. Based on these measurements, the spanwise components of the power spectral density and the correlation length of the turbulence were determined using a simple model for homogeneous and isotropic turbulence. The results of the study showed that the generated leading edge noise is nearly independent of angle of attack, only slightly dependent on camber but shows significant dependence on the thickness of the airfoil.

Staubs [11] performed an experimental study on the leading edge noise generated by three symmetric and two nonsymmetric airfoils that were subject to the turbulence generated by two grids, a square bar grid and a round bar grid. The parameters of the turbulent flow were measured, without the airfoil present, using a single-wire hot-wire 
probe. The leading edge noise was found to scale with the fifth power of the flow speed.

More recently, Hutcheson et al. [12] performed a detailed study on airfoil leading edge noise, examining the effect of the parameters of the incident turbulence (turbulence intensity, integral length scale), airfoil geometry (thickness and chord length), angle of attack and flow speed on the generation of leading edge noise at different model airfoils and flat plates. The homogeneous and nearly anisotropic turbulence was generated using two different grids, leading to turbulence intensities in the order of $5 \%$ to $8 \%$. The turbulence characteristics were measured using two-component hot-wire technique. Acoustic measurements were performed using thirteen microphones positioned at different elevation angles in the midspan plane of the airfoils in the acoustic far field. Additionally, qualitative microphone array measurements were performed on selected configurations to make sure that no noise was contributed from the junction of the airfoil models with the walls of the test section.

This brief review shows that several experimental studies on airfoil leading edge noise were performed in the past, most of them relying on single microphone measurements. Only few recent studies made use of microphone arrays to examine the location and, in some cases, the strength, of the noise sources at the leading edge of airfoils or flat plates. It also becomes apparent that, besides acoustic measurements, the measurement of characteristic parameters of the incident turbulence is essentially important to understand the mechanisms responsible for the noise generation at the leading edge.

The present paper describes in detail the measurement of the noise generated by the interaction of grid-generated turbulence with an airfoil leading edge in a subsonic flow using microphone array measurement technique. To allow for a noise source localization as exact as possible in order to separate the noise generated at the airfoil leading edge from that generated by the turbulence grids, a deconvolution beamforming algorithm is used which is extended to enable a three-dimensional mapping of noise source locations. Additional attention is given to the measurement of grid generated turbulence using hot-wire anemometry. As an example for the application of the selected beamforming algorithm, results of the measurement of the noise generated at the leading edge of three porous airfoils and one nonporous airfoil are presented briefly. The measured leading edge noise of the nonporous reference airfoil is compared to predictions using the model by Amiet [5].

\section{Experimental Setup}

Acoustic measurements were performed on a set of porous airfoils and a nonporous reference airfoil at zero angle of attack and subsonic flow speeds in a small aeroacoustic wind tunnel at the Brandenburg University of Technology in Cottbus. The inflow turbulence required for the generation of airfoil leading edge noise was provided by grids mounted to the nozzle exit of the open jet wind tunnel. Besides

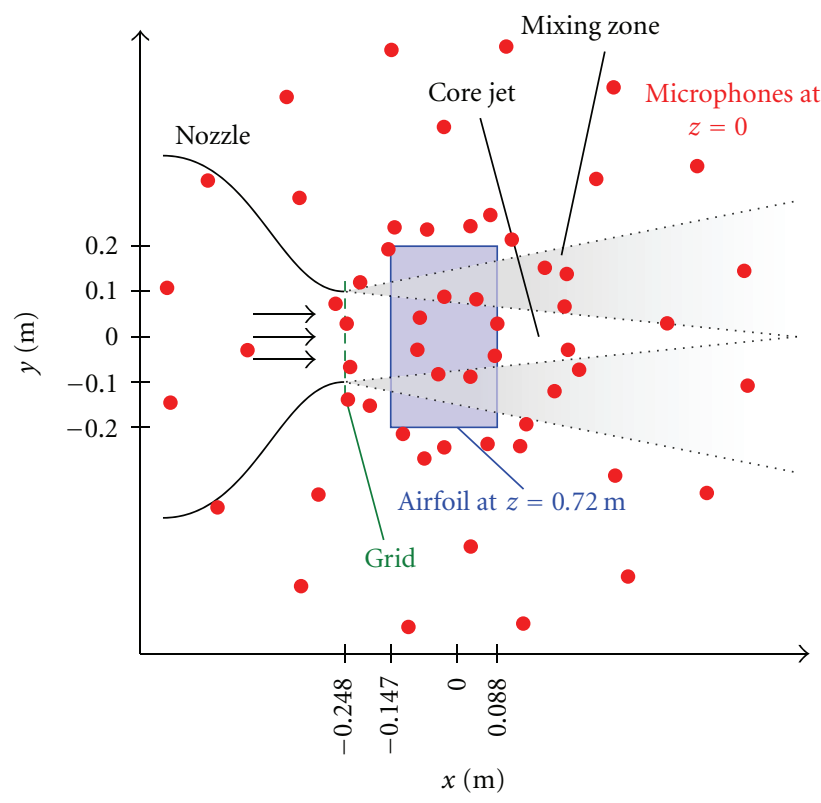

FIgURE 1: Schematic display of the measurement setup (top view).

the use of different turbulence grids, the distance between the grids and the airfoil leading edge position can be varied, thus providing a larger range of different incident turbulence parameters. These parameters were measured using hot-wire anemometry. The acoustic measurements were performed using a planar microphone array and deconvolution beamforming algorithms applied to a threedimensional source region. A schematic of the setup for the acoustic measurements is given in Figure 1.

2.1. Wind Tunnel. The aeroacoustic wind tunnel at Brandenburg University of Technology is an open jet wind tunnel [14]. For the present investigation, a circular Witoszynskitype nozzle with an exit diameter of $0.2 \mathrm{~m}$ was used. With this nozzle, the aeroacoustic wind tunnel has a very low turbulence in the core jet, which is in the order of $0.1 \%$ directly in front of the nozzle at a flow speed of $20 \mathrm{~m} / \mathrm{s}$. The wind tunnel self noise is also very low, with an overall sound pressure level below $60 \mathrm{~dB}(\mathrm{~A})$ for flow speeds up to $50 \mathrm{~m} / \mathrm{s}$, measured at $1 \mathrm{~m}$ distance at an angle of $90^{\circ}$ to the nozzle axis.

During acoustic measurements, the test section in front of the nozzle is surrounded by a cabin whose floor and side walls are equipped with a porous absorber, thus providing a nearly anechoic acoustic environment for frequencies above $500 \mathrm{~Hz}$. The planar microphone array used for the acoustic measurements forms the (hard-walled) ceiling of the cabin. The wall on the opposite side of the nozzle is open. Figure 2 shows a photograph of the setup (the nozzle is additionally equipped with an open-porous foam to avoid sound reflection effects). Note that throughout the present paper, the $x$-coordinate refers to the streamwise (chordwise) direction, the $y$-coordinate refers to the lateral (spanwise) direction, and the $z$-coordinate refers to the vertical direction. 


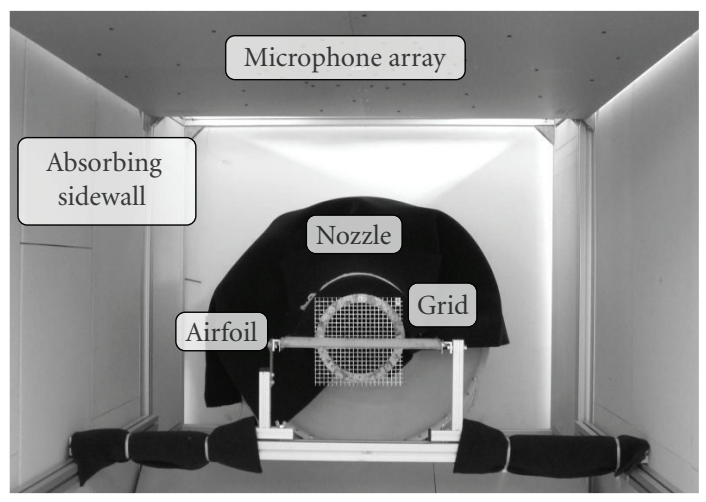

Figure 2: Photograph of the measurement setup, taken from downstream.

2.2. Turbulence Grids. Noise is generated at the leading edge of an airfoil if the incoming flow contains considerable turbulence. Possible means to generate this inflow turbulence are the use of cylinders or grids. In the present experiments, the turbulence was generated by two perforated plates with square holes, the geometry of which is defined by the parameters shown in Figure 3. Additionally, the parameter $t$ describes the thickness of the grids and the grid porosity $\beta$ is the ratio of the open area to the total area of the grid. The geometry parameters of both perforated plates are given in Table 1.

The turbulence grids were selected from a total of twelve available grids based on prior hot-wire measurements. The aim of these measurements was to identify those grids that generate a strong turbulence, and hence a high turbulence intensity

$$
T u=\frac{u_{\mathrm{rms}}}{U}=\frac{\sqrt{\overline{u^{2}}}}{U},
$$

at the position of the airfoil leading edge. In (2), $u_{\mathrm{rms}}$ is the root mean square of the turbulent velocity fluctuations and $U$ is the mean flow speed.

Another common parameter to characterize a turbulent flow, besides the turbulence intensity, is the integral length scale $\Lambda$ of the turbulence, which is a measure for the characteristic size of turbulent eddies within the flow. In the present study, the streamwise integral length scale was determined using the autocorrelation method based on the measurements with a single hot-wire probe only, according to

$$
\Lambda_{x}=U \int_{0}^{\infty} R(\tau) d \tau .
$$

The term $R(\tau)$ denotes the normalized autocorrelation of the velocity time series $u(t)$, with the offset (the mean flow velocity $U$ ) removed,

$$
R(\tau)=\frac{\overline{u(t) \cdot u(t-\tau)}}{\overline{u^{2}}},
$$

where the overline denotes the time mean. The calculation of such a scale using (3) and (4) is based on Taylors "frozen turbulence" hypothesis [15].



Figure 3: Definition of grid parameters: mesh width $M$, bar width or rod diameter $a$, and hole diameter $b$.

TABLE 1: Turbulence grids used in the experiments, grid parameters according to Figure 3 for perforated plates with square holes (PPS).

\begin{tabular}{lcccc}
\hline Abbr. & $\begin{array}{c}\text { Mesh width } \\
M[\mathrm{~mm}]\end{array}$ & $\begin{array}{c}\text { Bar width } \\
a[\mathrm{~mm}]\end{array}$ & $\begin{array}{c}\text { Thickness } \\
t[\mathrm{~mm}]\end{array}$ & $\begin{array}{c}\text { Porosity } \\
\beta\end{array}$ \\
\hline PPS $12 / 2$ & 12 & 2 & 1 & 0.69 \\
PPS $14 / 4$ & 14 & 4 & 1 & 0.51 \\
\hline
\end{tabular}

Since, according to the Wiener-Khinchine theorem [16], the autocorrelation function in the time domain corresponds to the autospectral density in the frequency domain, $R(\tau)$ may also be calculated in the frequency domain. This results in a noticeable reduction of computation time and easily allows for additional filtering of the signal. Thus, for the present study, the data were transformed into the frequency domain by using a Fast Fourier Transformation (FFT) on blocks with a size of 4,096 samples. The resulting frequency domain data were averaged over all blocks with an overlap of $50 \%$ and the autospectral density was calculated. The result was then transformed back into the time domain by using the Inverse Fast Fourier Transformation (IFFT). The integration in (3) was not performed over the entire available time domain, but from zero to the first zero-crossing as proposed by Katul and Parlange [17] and recommended by O'Neill et al. [18].

It has to be noted that the mean flow velocity at a certain distance downstream of a grid may vary from the mean flow velocity at a sufficiently large distance from the grid. Especially within an initial distance approximately ten mesh widths from the grid, the flow can be assumed to be inhomogeneous $[19,20]$. This is a result of the single wakes from each bar of the grid that are first isolated but then grow in size and finally merge into a homogeneous turbulent flow [19]. In the present paper, $U$ denotes the mean flow velocity dependent on the particular measurement position as is used for the calculation of $T u$ and $\Lambda_{x}$ according to (2) and (3), respectively. On the other hand, $U_{0}$ denotes the nominal flow speed, defined as the mean flow velocity at a larger distance to the grid where the turbulence is homogeneous. The nominal flow speed $U_{0}$ was determined in prior measurements at $x \approx 0.053 \mathrm{~m}$, and hence at a relatively large distance of $0.3 \mathrm{~m}$ from each grid, as a function of the pressure within the wind tunnel settling chamber. By adjusting the wind tunnel pressure, the nominal flow speed $U_{0}$ may be adjusted for each grid as a measure of the working point. 
TABLE 2: Airfoil models used in the experiments (all airfoils have a chord length of $0.235 \mathrm{~m}$ and a span width of approximately $0.4 \mathrm{~m}$ ).

\begin{tabular}{lcc}
\hline Name & Material & $r\left[\right.$ Pa s/m $\left.\mathrm{m}^{2}\right]$ \\
\hline Reference & Nonporous & $\infty$ \\
Needlona felt SO 2002 & Synthetic felt & 130,200 \\
Recemat & Metal foam & 8,200 \\
M-Pore Al 45 ppi & Metal foam & 1,000 \\
\hline
\end{tabular}

For acoustic experiments on airfoil leading edge noise by using grids to generate the inflow turbulence, basically two needs have to be met. On one hand, for the essential separation of noise generated by the grid itself from the noise sources located at the airfoil leading edge using microphone array technology, it is advantageous when the distance between the grid and the airfoil leading edge is not too small. Additionally, as mentioned above, grid generated turbulence can be assumed to be homogeneous and nearly isotropic at distances more than approximately ten mesh widths downstream of the grid. On the other hand, the turbulence intensity decreases with increasing distance from the grid. Therefore, the distance between the grid and the leading edge should not be too large; otherwise, the turbulence intensity at the position of the leading edge is lower and less leading edge noise is generated.

It was finally decided to perform measurements at several distances between grid and airfoil leading edge. In the present paper, however, acoustic results will be shown for the minimum distance only, which is one-half nozzle diameter $(0.1 \mathrm{~m})$ as indicated in Figure 1. At this distance, the turbulence generated by the two grids from Table 1 is possibly inhomogeneous.

2.3. Airfoil Models. Measurements were conducted on different porous airfoils and one nonporous reference airfoil with a chord length of $0.235 \mathrm{~m}$ and a span width of approximately $0.4 \mathrm{~m}$. The airfoils have a semisymmetric SD7003 shape designed for low Reynolds numbers. In order to enable the manufacturing of the porous airfoils out of the different porous materials, they have a slightly increased trailing edge thickness of $1.59 \mathrm{~mm}$ compared to $0.5 \mathrm{~mm}$ for the nonporous reference airfoil. Due to a past study on airfoil trailing edge noise $[21,22]$, the reference airfoil is additionally equipped with a thin tripping tape at $10.6 \%$ of the chord, which was not removed for the present experiments. In accordance to the findings by Oerlemans and Migliore [9], it is reasonable to assume that the tripping device has no influence on the generation of leading edge noise. Regarding the shape and dimension of the leading edge, the porous airfoils and the reference airfoil are identical.

The porous airfoils are characterized by their air flow resistivity $r$ [23], a material parameter that can be calculated based on the pressure difference $\Delta p_{s}$ across a cylindrical porous sample of cross-sectional area $A_{s}$ and thickness $d_{s}$ and the product of the velocity $u_{s}$ of a static fluid flow through the sample and the thickness:

$$
r=\frac{\Delta p_{s}}{u_{s} \cdot d_{s}}
$$

The air flow resistivity of the four different airfoils used in the present paper for the demonstration of the measurement techniques is given in Table 2 .

2.4. Microphone Array and Data Processing. The acoustic measurements were performed using a planar microphone array, which consists of $561 / 4$ inch microphone capsules flush-mounted into a $1.5 \mathrm{~m} \times 1.5 \mathrm{~m}$ aluminum plate. It has an aperture of $1.3 \mathrm{~m}$. The position of each microphone within the array is indicated in Figure 1. The microphone array is mounted out of the flow, at a distance of $0.72 \mathrm{~m}$ above the airfoil. For the demonstration of the acoustic measurement technique in the present paper, the streamwise position of the airfoil leading edge corresponds to $x=$ $-0.147 \mathrm{~m}$ in array coordinates ( $0.1 \mathrm{~m}$ from the nozzle).

The acoustic measurements were performed with a sample rate of $51.2 \mathrm{kHz}$ and a measurement duration of $40 \mathrm{~s}$, leading to a total of 2,048,000 samples per measurement. The raw data were stored and then further processed using deconvolution beamforming algorithms. In a first step, the data were blockwise transformed using an FFT with a Hanning window, each block having a size of 4,096 samples. This leads to a frequency spacing of $12.5 \mathrm{~Hz}$. The cross spectral matrix was calculated for each block and averaged over a total of 999 blocks with 50\% overlap.

During the acoustic measurements, the temperature in the test room was recorded and then used to correct the speed of sound in the subsequent data analysis.

When acoustic measurements are performed with a phased array of microphones, as opposed to measurements using only a single microphone, beamforming is the basic step of the postprocessing that focusses (or "steers") the array to different locations. In conventional beamforming, the noise sources located by the beamforming algorithm are usually mapped onto a two-dimensional plane (the result is commonly called a sound map). In the case of a planar microphone array, this two-dimensional plane is most often orientated parallel to the array. The source region then is represented by a two-dimensional grid, with potential noise sources assumed to be located at the grid points. For the present investigation, another approach was used, where potential sound sources are not assumed to be located within a planar source region only, but within a fully three-dimensional source region. This source region is then represented by a three-dimensional grid, and noise sources may be located at each point of this grid. The result of this beamforming technique is a three-dimensional distribution of source locations and the respective contributions to the sound pressure level as measured at the microphone array center. 
The complete source region analyzed in the present experiments has an extent of $0.6 \mathrm{~m}$ in the streamwise direction, $0.6 \mathrm{~m}$ in the spanwise direction, and $0.6 \mathrm{~m}$ in the vertical direction. The grid increment is $0.01 \mathrm{~m}$, resulting in a total of 226,981 grid points. The source region contains all potential noise source locations, including the airfoil leading edge and trailing edge, the wind tunnel nozzle with the turbulence grid, and the mountings at the sides of the airfoil.

When applying beamforming to a three-dimensional source region, the choice of the steering vector becomes very important. In general, different formulations of the steering vector are known for the analysis of microphone array measurements. In the present case, the formulation of the steering vector, which was for example also used by Dougherty [24] and Suzuki [25], is chosen.

In aeroacoustic microphone array measurements, it has to be taken into account that the results may be influenced by coherence loss effects, meaning the loss of coherence between single signals due to the propagation of the sound waves from the source to the microphones. These unwanted effects may occur when sound is scattered by turbulence, for example at the wind tunnel shear layer. Coherence loss is known to increase with increasing distance between the microphones within the array, with increasing flow speed and increasing frequency [26]. The potential influence of coherence loss effects on the present measurements was examined by using a relatively simple approach. According to Sijtsma and Stoker [27] the presence of coherence loss can be tested by comparing an acoustic image obtained by the entire microphone array to one obtained by a microphone array with the outer microphones excluded from the processing. If coherence loss is present, it will affect mainly these outer microphones. Thus, the exclusion of the outer microphones would actually lead to a higher resolution, and hence an increase of the peak levels and a decrease of the noise levels. If no coherence loss effects are present, the reduction of array aperture would result in a lower resolution.

The potential influence of coherence loss on the present measurements was examined by comparing the results of the original 56 microphone array geometry with those obtained with a reduced array geometry, consisting of only the 40 inner microphones (the outer circle of 16 microphones, see Figure 1, was excluded). The comparison of two-dimensional third-octave band sound maps calculated with a conventional delay-and-sum beamformer with diagonal removal [28] for both array geometries showed that coherence loss effects are negligible in the present case. The differences in absolute level and resolution are very small, even at the highest third-octave bands (with center frequencies of $16 \mathrm{kHz}$ and $20 \mathrm{kHz}$ ). The exclusion of the outer 16 microphones did not lead to a noticeable increase in peak level and a decrease in noise. Especially in the range of low frequencies, which is of interest in the present study, the 56 channel microphone array is not affected by coherence loss.

Different deconvolution algorithms were considered for the investigation of airfoil leading edge noise, including the CLEAN-SC algorithm proposed by Sijtsma [13] and the orthogonal beamforming (OB) algorithm proposed by Sarradj [29]. The CLEAN-SC deconvolution algorithm uses the spatial coherence between sources in the sound map and their side lobes and thus iteratively removes secondary coherent sound source components. The $\mathrm{OB}$ algorithm is based on an eigenvalue decomposition of the cross-spectral matrix of the microphone signals and uses the eigenvalues to estimate the absolute source levels and the corresponding eigenvectors to derive the source location. The DAMAS algorithm developed by Brooks and Humphreys [30], which uses a special iterative Gauss-Seidel technique to remove the convolution of the image of the sound sources with a theoretical point spread function, was not applied to the data. It was found to be computationally too expensive when used on a three-dimensional source region with an adequate resolution.

Past trailing edge noise measurements [21, 22] revealed that the CLEAN-SC algorithm delivers good results especially at low frequencies, while at high frequencies the CLEAN-SC may fail to deliver correct amplitudes of the located noise sources or may fail to locate noise sources at all. In the present study, the low frequency range of the noise sources located at the airfoil leading edge is of main interest, and thus it was decided to use the CLEAN-SC algorithm.

Figure 4 shows three-dimensional mappings of noise source locations (three-dimensional sound maps) with center frequencies of $2 \mathrm{kHz}$ and $6.3 \mathrm{kHz}$, obtained with the CLEAN-SC algorithm, for a measurement on the reference airfoil with the leading edge at $x=-0.147 \mathrm{~m}(0.1 \mathrm{~m}$ from the nozzle), downstream of the turbulence grid PPS $12 / 2$ at a nominal flow speed of approximately $45 \mathrm{~m} / \mathrm{s}$. Likewise, Figure 5 shows similar sound maps for the $2 \mathrm{kHz}$ thirdoctave band, but for the case without an airfoil and hence only the turbulence grid PPS 12/2 (the position of the airfoil is indicated in Figure 5 only to allow for comparison with Figure 4).

The figures illustrate that, when the airfoil is immersed in the turbulent flow generated by the grid, the resulting major noise sources at low frequencies (as visible for the $2 \mathrm{kHz}$ third-octave band) are located at the airfoil leading edge (Figures 4(a) and 4(b)), while at high frequencies $(6.3 \mathrm{kHz}$ third-octave band) the main sources are located at the turbulence grid (at $x=-0.248 \mathrm{~m}$, Figures $4(\mathrm{c}$ ) and $4(\mathrm{~d})$ ). Additionally, some weaker noise sources are visible for the low frequency case which are located at the position where the wind tunnel shear layer interacts with the airfoil trailing edge. If no airfoil is present, noise sources are located at the turbulence grid also in the range of low frequencies, as can be seen from Figure 5 .

Noise sources due to the interaction of an incident turbulence with the leading edge of an airfoil are considered to be generated by pressure fluctuations on the surface, and hence it would be assumed that the sources are located on the airfoil surface (or at least within a distance equal to a fraction of the corresponding acoustic wavelength from the surface). However, it can be seen from Figures 4(a) and 4(b) that the leading edge noise sources are noticeably spread in the vertical direction. The reason for this spreading is not completely clear yet. Possible reasons are random distance errors in the direction towards the array, the assumption of monopole sound sources in the underlying beamforming 


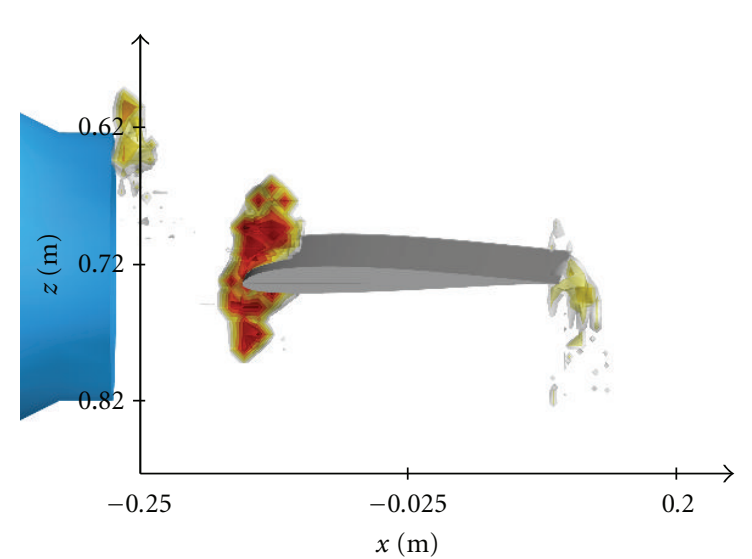

(a) $f_{c}=2 \mathrm{kHz}$, side view

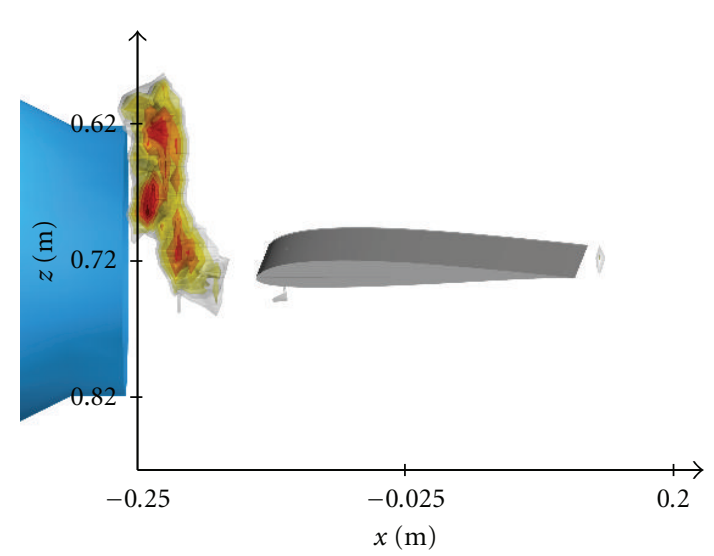

(c) $f_{c}=6.3 \mathrm{kHz}$, side view

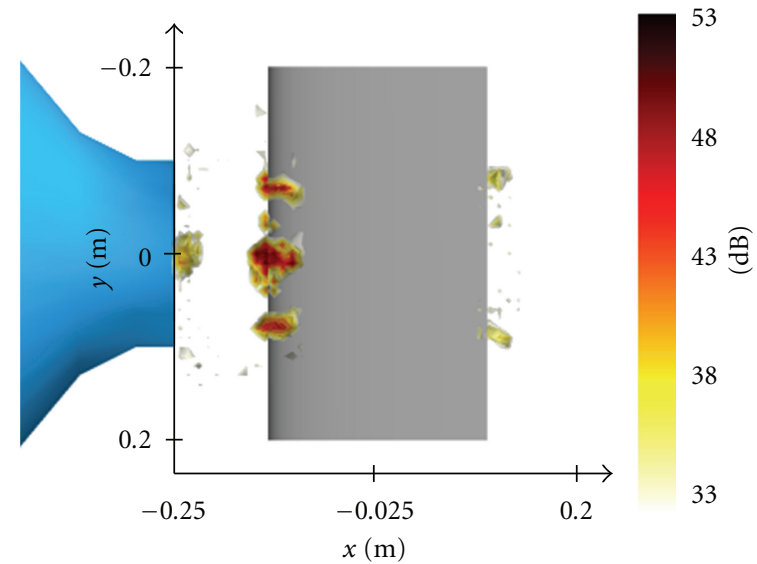

(b) $f_{c}=2 \mathrm{kHz}$, top view

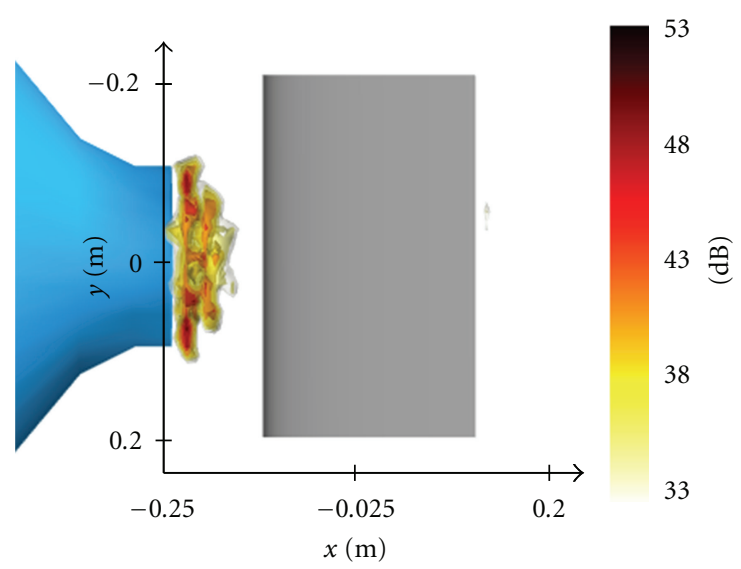

(d) $f_{c}=6.3 \mathrm{kHz}$, top view

FIGURE 4: Three-dimensional CLEAN-SC [13] sound maps obtained for the nonporous reference airfoil (view from above, flow from left to right, $U_{0} \approx 45 \mathrm{~m} / \mathrm{s}$, PPS $12 / 2$ turbulence grid-position not indicated-mounted directly to the nozzle exit).

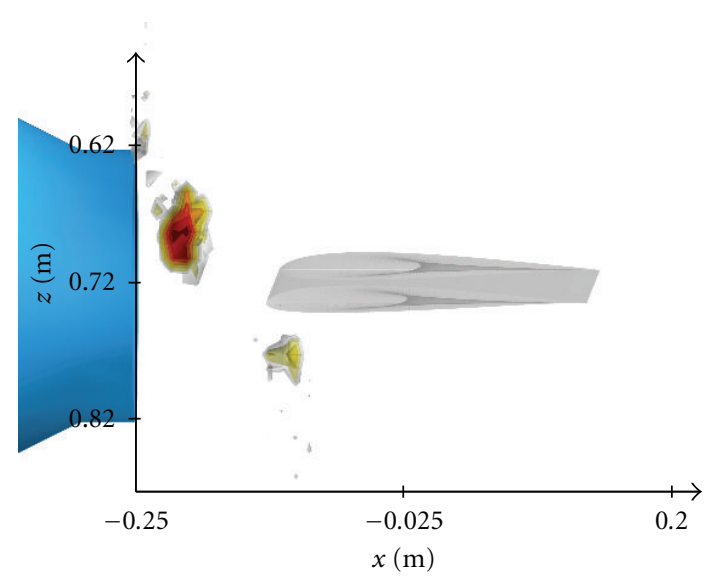

(a) $f_{c}=2 \mathrm{kHz}$, side view

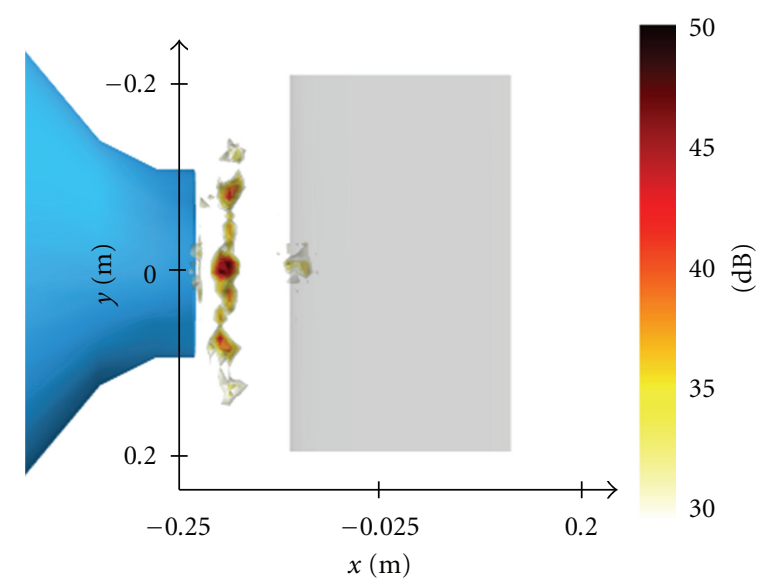

(b) $f_{c}=2 \mathrm{kHz}$, top view

FIgURE 5: Three-dimensional CLEAN-SC [13] sound maps obtained for the empty test section (position of the airfoil is indicated to enable comparison with Figure 4) (view from above, flow from left to right, $U_{0} \approx 45 \mathrm{~m} / \mathrm{s}$, PPS 12/2 turbulence grid-position not indicatedmounted directly to the nozzle exit). 
algorithm, and the presentation of third-octave band results (as opposed to results at a single frequency, where no spreading can be noticed, but where the sources are also not necessarily positioned at the airfoil surface). Another reason may be that, although it is a common assumption, the leading edge noise is not only generated directly at the surface of the airfoil, but also within a certain distance from the surface. It also has to be kept in mind that a two-dimensional visualization of three-dimensional sound sources in general is difficult to interpret.

To obtain spectra of the noise generated at the leading edge of the airfoils, the noise source contributions are integrated over a three-dimensional volume (as opposed to a two-dimensional sector as used in two-dimensional beamforming) that contains only the airfoil leading edge, but no other potential noise source location. Major noise sources that are excluded from the integration are sources located at the turbulence grid (grid self-noise), noise sources that are generated due to the impingement of the wind tunnel shear layer on the airfoil leading edge and surface as well as noise sources located at the airfoil trailing edge. The chosen volume, shown in Figure 6, is cubic and has a side length of $0.1 \mathrm{~m}$. It is located completely within the wind tunnel core jet and therefore not affected by interactions of the shear layer with the airfoil leading edge. For a leading edge position of $x=-0.147 \mathrm{~m}$ (and hence a distance between turbulence grid and leading edge of $0.1 \mathrm{~m}$ ) as used for the present demonstration, the resulting boundaries of the leading edge noise volume are $-0.167 \mathrm{~m} \leq x \leq-0.067 \mathrm{~m}$ (streamwise direction), $-0.05 \mathrm{~m} \leq y \leq 0.05 \mathrm{~m}$ (spanwise direction), and $0.67 \mathrm{~m} \leq z \leq 0.77 \mathrm{~m}$ (vertical direction).

For reasons of comparison, the spectra resulting from the integration over the leading edge volume for the case with the airfoil removed (empty test section) will also be considered in the analysis of the measurements, although the results are not physically meaningful since no distinct noise sources are located within this volume. However, they will be included as an approximate measure of the background noise.

The examination of the three-dimensional sound maps indicates that care has to be taken regarding the frequency range to be analyzed, since at high frequencies background noise may be present within the airfoil leading edge noise sector. Besides this potential upper frequency limit, acoustic measurements on airfoil edge noise, referring to either leading edge or trailing edge, are usually constricted to the acoustic frequency range at which the corresponding wavelengths are small compared to the chord length of the airfoil. In that case the noncompactness condition [31], $\lambda<$ $c_{l}$, is met, which in the present experimental study holds true for frequencies approximately equal to and larger than $1.5 \mathrm{kHz}$. Above this frequency, the noise generated by the airfoil leading edge can clearly be considered as separated from the noise generated at the trailing edge. Although the use of microphone array technology should allow for the identification and separation of edge noise sources at frequencies somewhat below that limit, the lowest thirdoctave band considered in the present analysis has a center frequency of $1.6 \mathrm{kHz}$.

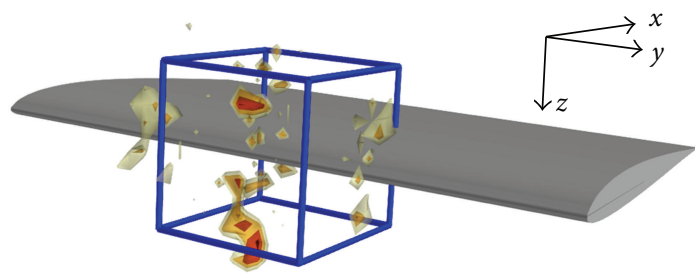

FIGURE 6: Chosen integration volume for airfoil leading edge noise (sample sound map: nonporous reference airfoil, PPS 12/2 turbulence grid, $U_{0} \approx 35 \mathrm{~m} / \mathrm{s}$, CLEAN-SC, $2 \mathrm{kHz}$ third-octave band).

No correction for the refraction of sound at the open jet shear layer was applied due to the fact that the exact shape and thickness of the conical shear layer of the wind tunnel is not known. Common correction procedures, like the method developed by Amiet and Schlinker [32, 33], are based on the assumption of a cylindrical shear layer of constant thickness. Such procedures do not seem appropriate for the present measurement setup and the necessary effort for the implementation does not seem justified. Methods employing a more sophisticated three-dimensional geometric correction, whose implementation is way more complex, do not necessarily lead to considerably better results [34]. Additionally, the distance between the noise source locations at the leading edge and the shear layer is relatively small (in the order of one half nozzle diameter, $0.1 \mathrm{~m}$ ) compared to the distance of $0.72 \mathrm{~m}$ between the leading edge and the microphone array center, which results in only small deviations of the noise source locations due to refraction at the shear layer.

However, a rough estimate for the effect of the shear layer refraction on the localization of the noise sources can easily be given based on common two-dimensional geometric corrections $[32,35]$. For a Mach number of 0.13 , corresponding to the maximum Mach number of the present investigation, the shift of the noise sources due to the shear layer is in the order of $0.01 \mathrm{~m}$ and is hence as small as the grid resolution of the present case.

Finally, the measured sound pressure levels were corrected for the reflection at the microphone array flat plate by subtracting $6 \mathrm{~dB}$.

2.5. Characterization of the Incident Turbulence. To examine if the presence of the airfoils has an influence on the flow field directly upstream of the leading edge, which may especially be of interest for airfoils with low air flow resistivities $r$ compared to the nonporous reference airfoil, prior hot-wire measurements were performed in a plane normal to the flow upstream of the airfoil leading edge.

A multichannel constant temperature anemometry (CTA) measurement system with a right-angled single-wire probe (Dantec 55P14) was used for these measurements. The probe, with the wire aligned with the spanwise axis, was positioned using a $3 \mathrm{D}$ traverse system with a minimum step size of $0.1 \mathrm{~mm}$. The measurement system contains a $10 \mathrm{kHz}$ low-pass filter. The velocity time series was recorded with a sample frequency of $25.6 \mathrm{kHz}$ and a measurement 
duration of $10 \mathrm{~s}$ using a 24 Bit National Instruments data acquisition system. To eliminate the influence of possible vibrations of the hot-wire probe after each step of the traverse system, the first-second of each measured data set was omitted, leaving 230,400 samples to be analyzed. With $50 \%$ overlap and a block size of 4,096 samples, this led to 112 blocks on which the FFT was performed and which were subsequently averaged. An additional $10 \mathrm{~Hz}$ high-pass filter was implemented in the analysis software in order to eliminate the offset voltage. All hot-wire calibrations were performed with the velocity calibration method using a Pitot tube.

The measurements were performed in a plane normal to the flow at $x=-0.154 \mathrm{~m}$ and hence at a distance of $0.007 \mathrm{~m}$ upstream of the leading edge of three different airfoils (the nonporous reference airfoil, the porous airfoil made of M-Pore Al $45 \mathrm{ppi}, r=1,000 \mathrm{~Pa} \mathrm{~s} / \mathrm{m}^{2}$, and the porous airfoil made of Recemat, $r=8,200 \mathrm{~Pa} \mathrm{~s} / \mathrm{m}^{2}$ ), successively using both turbulence grids from Table 1. The distance of $0.007 \mathrm{~m}$ was the smallest possible spacing due to the length of the prongs of the hot-wire probe. Each plane consists of 416 single-measurement points for the measurements upstream of the non-porous airfoil and the airfoil made of M-Pore Al 45 ppi and 312 points for the measurements upstream of the Recemat airfoil. The aim of these prior hot-wire measurements was to determine the number of measurement points which is necessary to properly capture the parameters of the turbulent inflow.

Figure 7 shows the root-mean-square of the turbulent velocity fluctuations $u_{\mathrm{rms}}=\sqrt{\overline{u^{2}}}$ and the mean flow velocity $U$ measured upstream of the three airfoils. For these measurements, the airfoil was positioned with the leading edge at $x=-0.147 \mathrm{~m}(0.1 \mathrm{~m}$ downstream of the turbulence grids). Thus, the hot-wire measurement plane was positioned at $x=-0.154 \mathrm{~m}(0.007 \mathrm{~m}$ upstream of the leading edge and $0.093 \mathrm{~m}$ downstream of the grid). As mentioned above, at this distance, which is slightly smaller than ten mesh widths for each grid, the generated turbulence cannot be expected to be fully homogeneous and anisotropic.

As suspected, Figure 7 reveals that the flow field and the turbulence generated at this distance is not homogeneous, but shows differences which are correlated to the geometry of the grids. At regions that correspond to holes in the grid, the rms velocity is larger than in the "shadowed" region behind the grid bars. However, Figure 7 also shows that the porous material only has a small influence on the flow parameters upstream of the leading edge. For the three different airfoils, there are only minor differences in the rms velocity and the mean flow speed. In order to determine the characteristic parameters of the incident turbulence with a sufficient statistical significance, the necessary number of measurements has to be further investigated.

Corresponding to the data shown in Figure 7, Table 3 lists the mean value $\bar{u}_{\text {rms }}$ and the standard deviation $\sigma_{u_{\mathrm{rms}}}$ obtained for the rms of the turbulent velocity fluctuations $u_{\mathrm{rms}}$ as well as the mean value $\bar{U}$ and the standard deviation $\sigma_{U}$ obtained for the mean flow speed $U$ for the three airfoils. Table 3 confirms the conclusion from Figure 7, namely, the
TABLE 3: Mean value (denoted by overline) and standard deviation $\sigma$ of both the rms of the turbulent velocity fluctuations $u_{\text {rms }}$ and the mean flow velocity $U$ of the measured data shown in Figure 7.

\begin{tabular}{lccccc}
\hline Grid & $\begin{array}{c}\text { Airfoil } \\
(r[\mathrm{~Pa} \mathrm{~s} / \mathrm{m} 2])\end{array}$ & $\begin{array}{c}\bar{u}_{\text {rms }} \\
{[\mathrm{m} / \mathrm{s}]}\end{array}$ & $\begin{array}{c}\sigma_{u_{\mathrm{rms}}} \\
{[\mathrm{m} / \mathrm{s}]}\end{array}$ & $\begin{array}{c}\bar{U} \\
{[\mathrm{~m} / \mathrm{s}]}\end{array}$ & $\begin{array}{c}\sigma_{U} \\
{[\mathrm{~m} / \mathrm{s}]}\end{array}$ \\
\hline PPS 12/2 & $\infty$ & 2.37 & 0.12 & 22.32 & 1.90 \\
& 1,000 & 2.42 & 0.11 & 23.36 & 1.32 \\
PPS 14/4 & 8,200 & 2.39 & 0.12 & 23.23 & 1.36 \\
& $\infty$ & 3.00 & 0.14 & 21.75 & 1.48 \\
& 1,000 & 3.07 & 0.11 & 22.88 & 0.95 \\
& 8,200 & 3.09 & 0.13 & 22.97 & 1.15 \\
\hline
\end{tabular}

influence of the materials air flow resistivity on the incident turbulence is negligible. It also shows that the standard deviation of the mean flow velocity and the rms velocity is relatively small.

Since the rms value of the streamwise velocity fluctuations $u_{\mathrm{rms}}$ is needed for both the calculation of the turbulence intensity according to (2) and the integral length scale according to (4), it is reasonable to identify $u_{\text {rms }}$ as the one crucial parameter characterizing the incident turbulence.

Based on Amiet's model in the notation given by (1) it can be further assumed that the far field intensity of airfoil leading edge noise depends on the square of the rms velocity fluctuations. If the difference in the sound pressure level $\Delta L_{p}$ resulting from variations of the measured rms velocity should remain less than, say, $1 \mathrm{~dB}$, the corresponding difference in rms velocity $\Delta u_{\mathrm{rms}}$ responsible for this sound pressure level difference can be determined. Then, under the assumption of a Gaussian distribution, the number of samples $m$ necessary to obtain $\bar{u}_{\text {rms }}$ within the $99 \%$ confidence interval for $\bar{u}_{\text {rms }}=\bar{u}_{m, \mathrm{rms}} \pm \sigma_{u_{m, \mathrm{rms}}}$ can be estimated [36]. Based on $\bar{u}_{\text {rms }}$ and $\sigma_{u_{\mathrm{rms}}}$ from Table 3 this led to the result that hot-wire measurements at only one single position would be necessary. This was also confirmed by examining the influence of the number of measurement points on the mean value of the rms velocity on the data from Figure 7. However, it was decided to perform hotwire measurements at $m=15$ positions upstream of the nonporous reference airfoil only.

Hence, for each of the grids used in the present study, CTA measurements were performed upstream of the nonporous airfoil, using a right-angled single-wire probe. The 15 measurement positions were randomly distributed within a plane normal to the flow and located at $x=-0.154 \mathrm{~m}$ ( $0.007 \mathrm{~m}$ upstream of the leading edge). The plane is centered around the leading edge at midspan and has a height of $0.034 \mathrm{~m}$ (and thus extends the maximum airfoil thickness) and width of $0.1 \mathrm{~m}$ (and thus remains inside the wind tunnel core jet).

The sample frequency of the hot-wire measurements was set to $25.6 \mathrm{kHz}$ with a measurement duration of $16 \mathrm{~s}$. As was done with the results from the prior hot-wire measurements, the first-second of the recorded data was omitted in order to avoid any potential influence of vibrations of the hotwire probe on the result, this time leaving 384,000 samples 

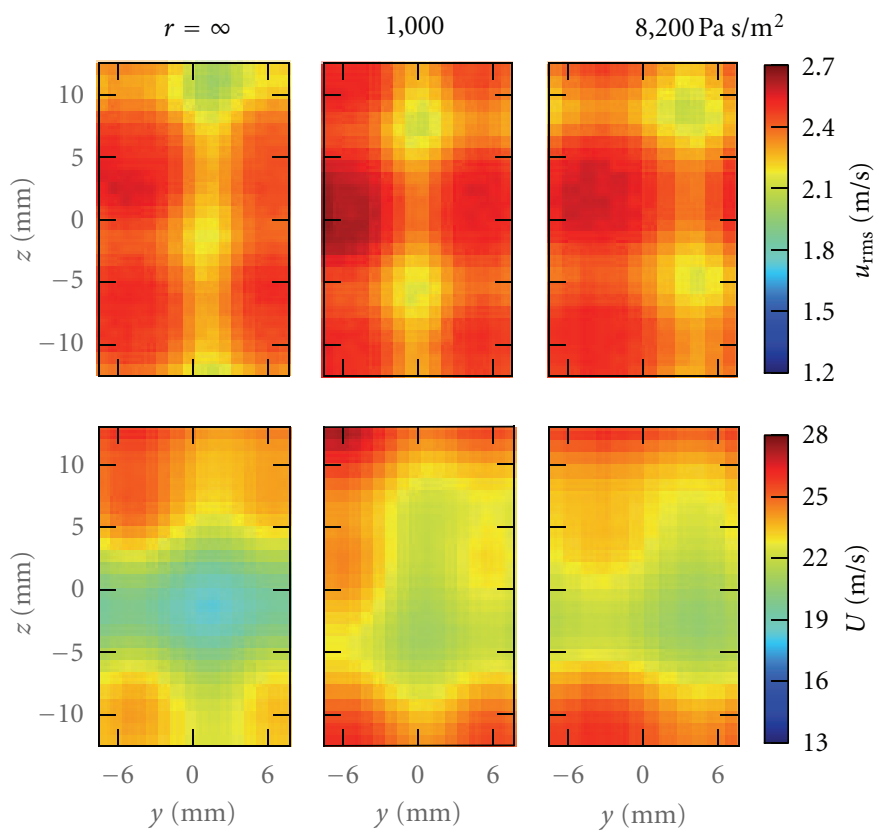

(a) PPS 12/2 turbulence grid
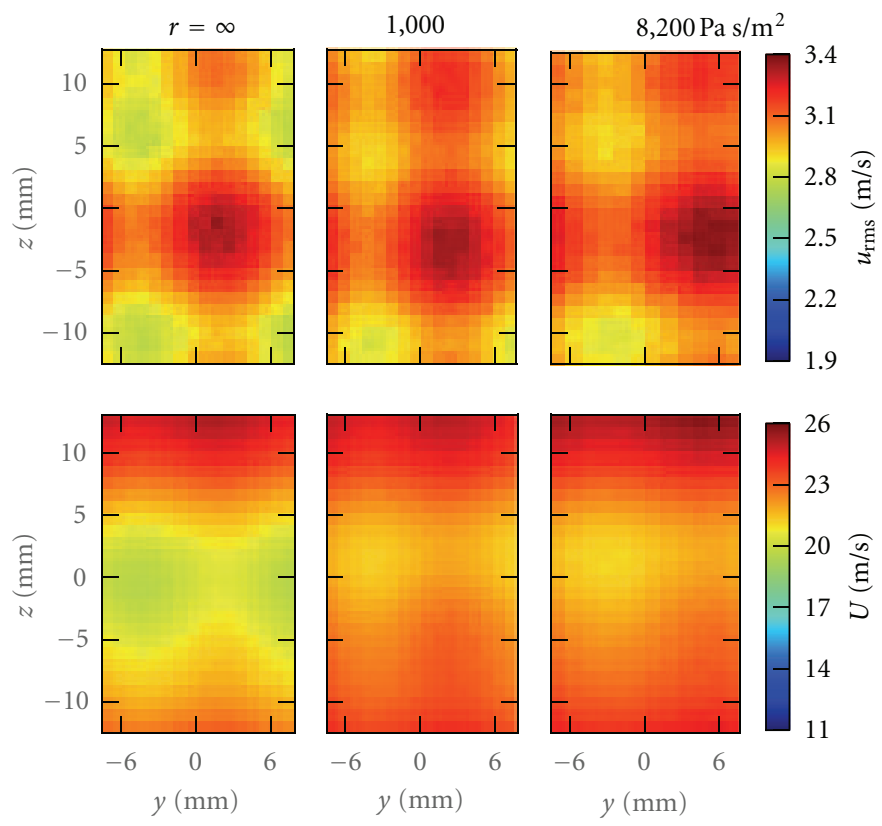

(b) PPS 14/4 turbulence grid

FIGURE 7: Results of preliminary CTA measurements to determine the possible influence of the presence of the airfoil on the flow parameters directly upstream of the leading edge, nominal flow speed $U_{0}=30 \mathrm{~m} / \mathrm{s}$, airfoil leading edge at $x=-0.147 \mathrm{~m}$ (distance between turbulence grid and leading edge $0.1 \mathrm{~m}$ ). Note that the dynamic range of the figures is small, $1.5 \mathrm{~m} / \mathrm{s} \mathrm{and} 15 \mathrm{~m} / \mathrm{s}$, respectively.

for the analysis. With a block size of 4,096 samples and an overlap of 50\%, this leads to 187 blocks that were Fast Fourier transformed into the frequency domain and then averaged.

In the present paper, instead of specifying the turbulence intensity according to (2), $u_{\text {rms }}$ will be directly used to characterize the intensity of the turbulent flow. Likewise, instead of calculating the streamwise integral length scale $\Lambda_{x}$ according to (3), the parameter

$$
t_{0}=\frac{\Lambda_{x}}{U}=\int_{0}^{\infty} R(\tau) d \tau
$$

will be used to characterize the size of the eddies. This parameter can be regarded as a measure corresponding to the time that a coherent turbulent structure (a turbulent eddy) needs to pass the hot-wire probe. The advantage of specifying $u_{\mathrm{rms}}$ and $t_{0}$ instead of $T u$ and $\Lambda_{x}$ is that the knowledge of the mean flow velocity $U$ is not required.

Figure 8 shows the parameters mean flow velocity $U$, rms of the velocity fluctuations $u_{\mathrm{rms}}$, and $t_{0}$ of the turbulent inflow generated by the turbulence grids from Table 1 , including the standard deviation due to the measurement at 


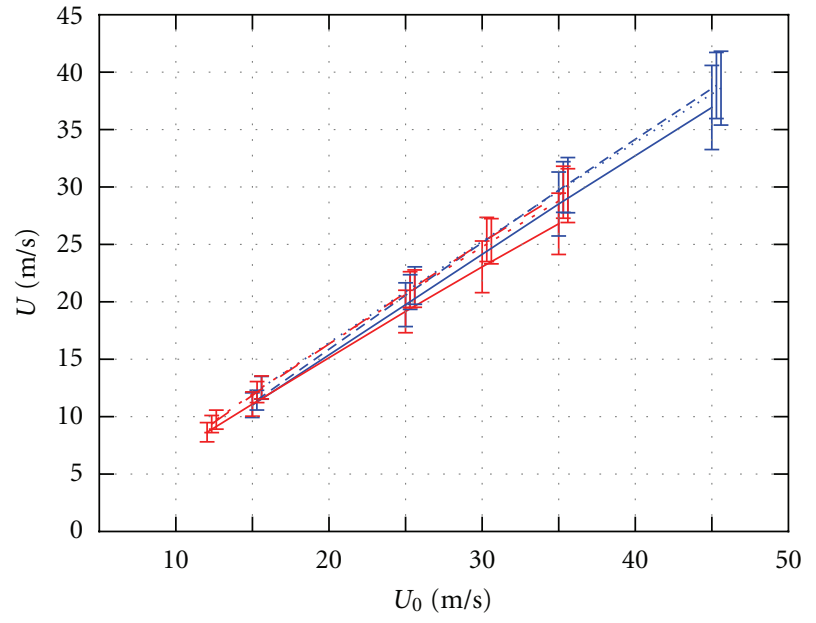

(a) Mean flow velocity

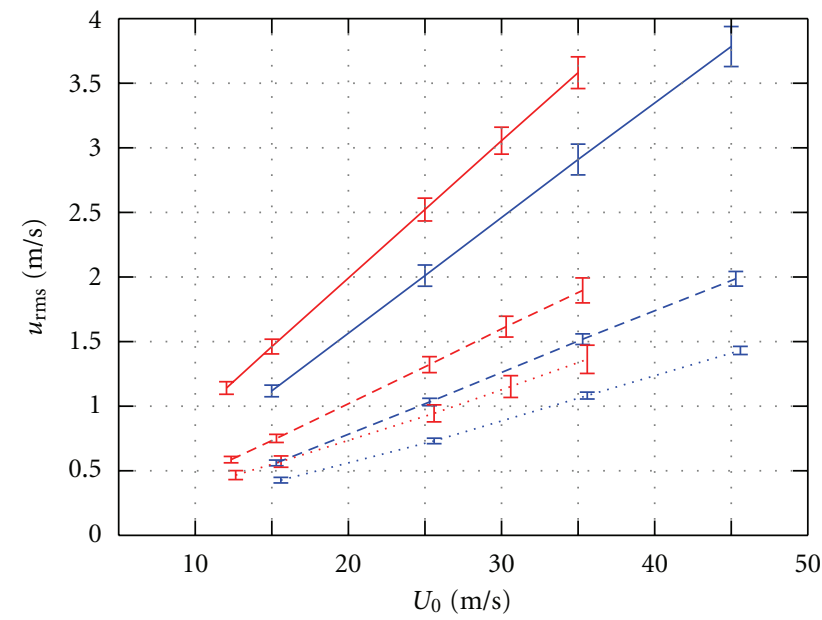

(b) Root-mean-square of the turbulent velocity fluctuations

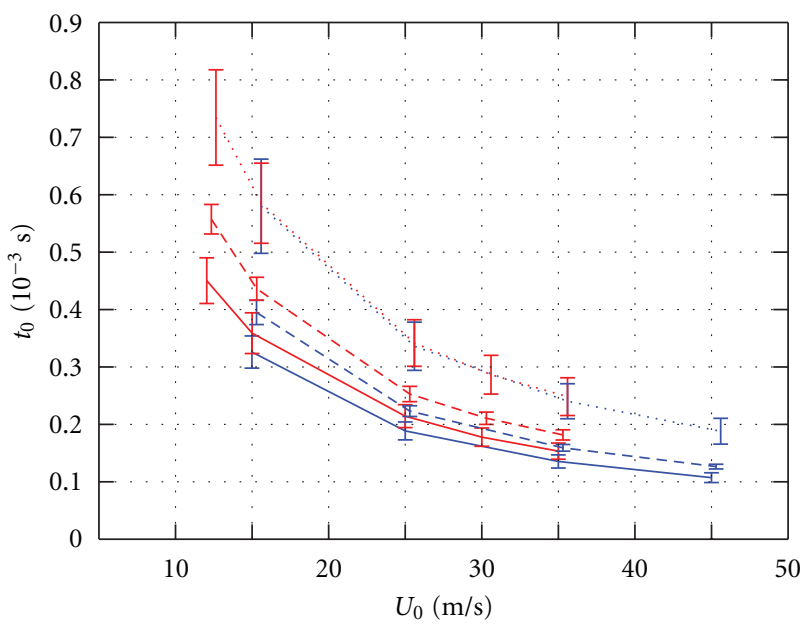

(c) $t_{0}$ according to $(6)$

FIGURE 8: Parameters of the turbulent inflow, measured in a plane $7 \mathrm{~mm}$ upstream of the leading edge of the nonporous reference airfoil, as a function of the nominal flow speed $U_{0}$ (turbulence grids: (blue-dash) PPS 12/2, (red-dash) PPS 14/4), solid lines: airfoil leading edge at $x=-0.147 \mathrm{~m}$ (distance to turbulence grid $0.1 \mathrm{~m}$ ), dashed line: $x=-0.047 \mathrm{~m}(0.2 \mathrm{~m})$, dotted line: $x=0.053 \mathrm{~m}(0.3 \mathrm{~m})$.

15 positions within the plane upstream of the nonporous reference airfoil, for three different distances between the grid and the airfoil leading edge.

Figure 8(a) shows that the mean flow velocity $U$ at the approximate position of the leading edge is lower than the nominal flow speed $U_{0}$ for both grids, which is due to the presence of the airfoil and the location of the measurement positions near the stagnation point. The different distances of the measurement plane from the turbulence grids only have a negligible influence on the mean flow velocity. As would be expected, the rms of the turbulent velocity fluctuations, $u_{\mathrm{rms}}$, increases for increasing nominal flow speed, as can be seen from Figure 8(b), while it decreases with increasing distance from the grid. In general, $u_{\mathrm{rms}}$ is higher for the second turbulence grid from Table 1, the PPS 14/4 grid. This is assumed to be due to the larger bar width of this grid compared to the PPS $12 / 2$ grid, which seems to be one of the main geometry parameters governing grid-generated turbulence. The parameter $t_{0}$, presented in Figure $8(\mathrm{c})$, decreases with increasing nominal flow speed. At positions of $x=-0.147 \mathrm{~m}$ and $x=-0.047 \mathrm{~m}$ (distances of $0.1 \mathrm{~m}$ and $0.2 \mathrm{~m}$ from the turbulence grids), $t_{0}$ is larger for the PPS 14/4 grid, while at the largest distance from the grid at $x=0.053 \mathrm{~m}$, it is similar for both grids. Judging from the standard deviations of the parameters shown in Figures 8(a) through $8(\mathrm{c})$, no noticeable differences can be found between the supposedly inhomogeneous turbulence at $x=-0.147 \mathrm{~m}$ and the turbulence at larger distances.

As a measure for the quality of the grid-generated turbulence, Figure 9 shows the power spectral density $\Phi$ of the turbulent velocity fluctuations (turbulence spectra). It was obtained at one single point within the hot-wire measurement plane upstream of the airfoil leading edge close to midspan. The measured turbulence spectra have a frequency step size of $6.25 \mathrm{~Hz}$ due to the aforementioned sample frequency and the block size used for the FFT. Therefore, the amplitude of the spectra given in Figure 9 was corrected to correspond to a frequency step size of $1 \mathrm{~Hz}$. 
It can be seen from Figure 9 that, while the spectral shape of the turbulence is generally similar for both grids, the turbulence generated by the PPS 14/4 grid has a higher energy in the complete range of frequencies. It was additionally found that the spectra agree well with the predictions given in [19] for homogeneous, isotropic turbulence when normalized with the mean velocity, the rms of the turbulent velocity fluctuations and the streamwise integral length scale.

\section{Results}

In total, measurements were conducted at zero angle of attack for different flow speeds between $10 \mathrm{~m} / \mathrm{s}$ and $60 \mathrm{~m} / \mathrm{s}$ (corresponding to Mach numbers $\mathrm{Ma}$ between approximately 0.03 and 0.17 and to chord based Reynolds numbers Re between approximately 157,000 and 940,000) for both turbulence grids from Table 1. In order to demonstrate the measurement technique and data processing, results will only be shown for one nominal flow speed of approximately $35 \mathrm{~m} / \mathrm{s}$.

3.1. Sound Maps. As a first result, Figures 10 and 11 show sample third-octave band sound maps for the three porous airfoils and the reference airfoil for a center frequency of $2 \mathrm{kHz}$, and hence for the frequency domain, where the airfoil leading edge is the dominating noise source. In Figure 10, the turbulence is generated by the PPS 12/2 grid and in Figure 11 it is generated by the PPS 14/4 grid.

In general, different noticeable noise sources are localized by the chosen beamforming algorithm. This includes two lateral sources due to the interaction of the wind tunnel shear layer with the leading edge and the source due to the interaction of the leading edge with the grid generated turbulence, located at the airfoil leading edge approximately at midspan. At the position of the turbulence grid, upstream of the airfoils and some distance above the airfoil leading edge, are the locations of weaker noise sources generated by the grids.

The turbulent flow generated by the PPS 14/4 grid leads to a considerably higher noise generation than the turbulent flow generated by the PPS $12 / 2$ grid. This is due to the basically higher turbulent kinetic energy, as observed in Figures 8 and 9 , supposedly caused by the thicker bars of the PPS $14 / 4$ grid.

It can be seen from Figures 10 and 11 that in most cases the noise generated at the leading edge of the porous airfoils at the $2 \mathrm{kHz}$ third-octave band is below that generated at the leading edge of the nonporous reference airfoil, the noise reduction being in the order of $1 \mathrm{~dB}$ to $2 \mathrm{~dB}$. For the measurement involving the PPS $12 / 2$ turbulence grid, the maximum of the noise generated by the porous airfoil made of Needlona felt $\left(r=130,200 \mathrm{~Pa} \mathrm{~s} / \mathrm{m}^{2}\right)$ exceeds the noise generated by the reference airfoil, while for the measurement with the PPS 14/4 grid the porous airfoil made of M-Pore Al $45 \mathrm{ppi}\left(r=1,000 \mathrm{~Pa} \mathrm{~s} / \mathrm{m}^{2}\right)$ generates a slightly higher peak value of the leading edge noise than the reference airfoil. However, the sound maps also indicate that the spatial extent of the noise sources at the leading edge midspan region of

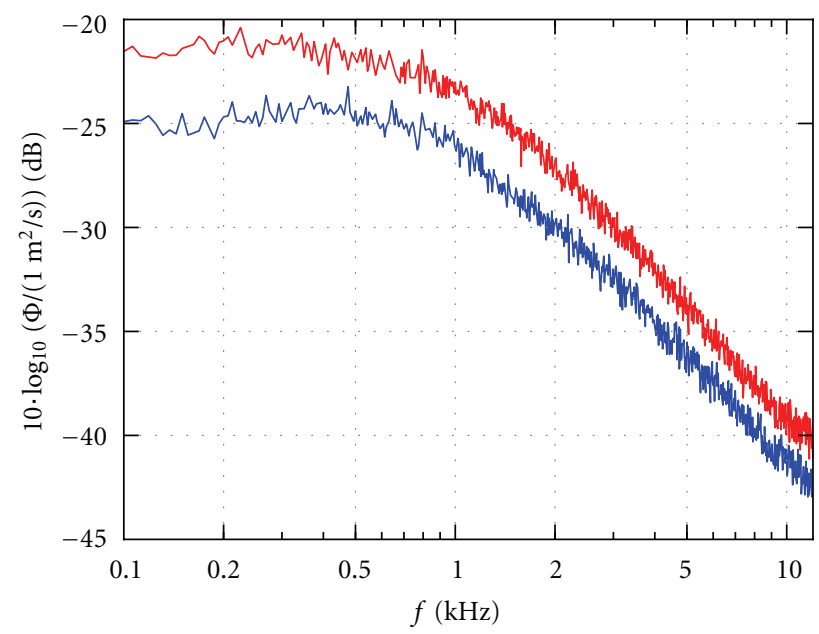

FIGURe 9: Power spectral density $\left(r e 1(\mathrm{~m} / \mathrm{s})^{2} / \mathrm{Hz}\right)$ of the turbulent velocity fluctuations, measured at a nominal flow speed $U_{0}=$ $35 \mathrm{~m} / \mathrm{s}$ approximately at $x=-0.154 \mathrm{~m}, 0.007 \mathrm{~m}$ upstream of the leading edge of the non-porous reference airfoil at mid-span (turbulence grids: (blue -) PPS 12/2, (red -) PPS 14/4).

the reference airfoil is larger than that of the sources at the porous airfoils.

The differences between the leading edge noise generated by the different airfoils seem very small only, judging from the sound maps shown in Figures 10 and 11, but it has to be noted that it is generally difficult to estimate the source strength from sound maps. To allow quantitative comparisons of the corresponding source strengths, sound pressure level spectra will be shown in the following section.

3.2. Sound Pressure Level Spectra. Third-octave band sound pressure level spectra that were obtained by integration of the three-dimensional sound maps over the leading edge noise volume shown in Figure 6 for a flow speed of $35 \mathrm{~m} / \mathrm{s}$ are shown in Figure 12(a) for the first turbulence grid from Table 1 and in Figure 12(b) for the second grid.

Additionally, each figure contains the leading edge noise spectrum calculated using the Amiet model [5] as given by (1), which employs the Karman model to describe the turbulence spectrum. The required input parameters for the model are the spanwise extent of the chosen leading edge noise sector, the distance between the array center and the leading edge at mid-span, the mean flow velocity $U$, the rms of the turbulent velocity fluctuations $u_{\mathrm{rms}}$, the streamwise integral length scale of the turbulence $\Lambda_{x}$ and the speed of sound $c$.

The leading edge noise predicted by the Amiet model is clearly higher than the leading edge noise measured for the nonporous reference airfoil. Depending on the frequency, the differences are in the order of $10 \mathrm{~dB}$ to $20 \mathrm{~dB}$. The inclusion of the airfoil thickness correction developed by Gershfeld [37] for the Amiet model did not lead to a better agreement in the range of frequencies examined. A possible reason for the large difference is that the high frequency solution of Amiet's model according to (1) was designed for Mach numbers which "are not too small," whereas the Mach number in the 




(a) Nonporous reference airfoil, $r=\infty$

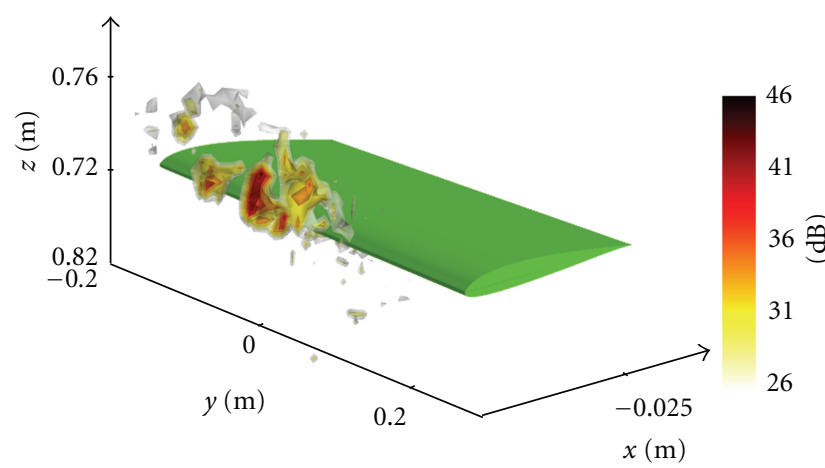

(c) Porous airfoil made of Recemat, $r=8,200 \mathrm{~Pa} \mathrm{~s} / \mathrm{m}^{2}$

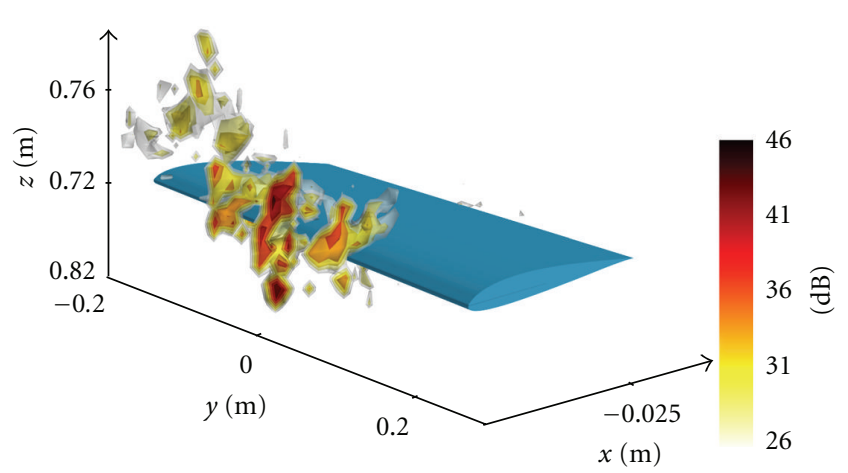

(b) Porous airfoil made of Needlona felt, $r=130,200 \mathrm{~Pa} \mathrm{~s} / \mathrm{m}^{2}$

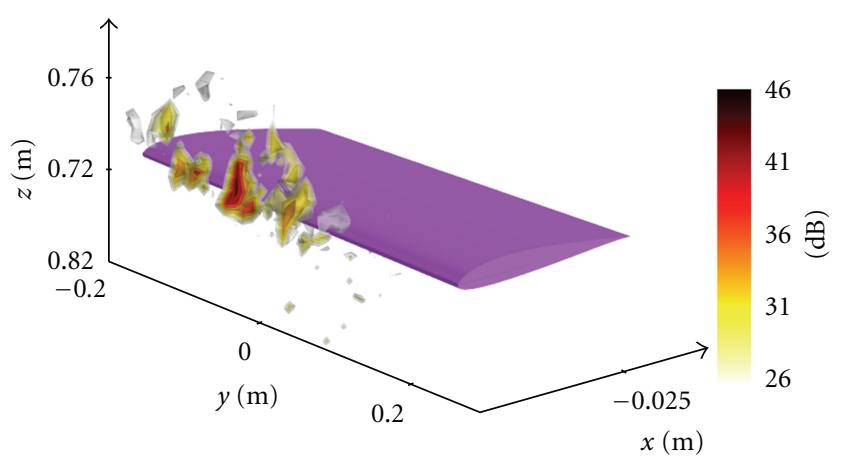

(d) Porous airfoil made of M-Pore Al 45 ppi, $r=1,000 \mathrm{~Pa} \mathrm{~s} / \mathrm{m} 2$

FIgure 10: Three-dimensional third-octave band sound maps, PPS 12/2 turbulence grid, $U_{0}=35 \mathrm{~m} / \mathrm{s}(\mathrm{Re}=548,000)$, CLEAN-SC beamforming algorithm, center frequency $2 \mathrm{kHz}$. (Note that all sound maps are scaled to the same maximum level measured for the reference airfoil.).

present experiments only takes a value of about 0.1 . Moreau and Roger [10] also observed a noticeable overestimation of the Amiet model at low flow speeds (corresponding to Mach numbers around 0.1) and high frequencies in comparison to leading edge noise spectra measured for a $0.1 \mathrm{~m}$ chord NACA 0012 airfoil. The comparison of the leading edge noise spectra measured by Staubs [11] on a $0.203 \mathrm{~m}$ chord NACA 0012 airfoil at Mach numbers of 0.087 and 0.117 also showed that the Amiet model seems to overpredict the noise. In the case of Staubs' results, the Amiet model gave third-octave band sound pressure levels approximately $2 \mathrm{~dB}$ to $3 \mathrm{~dB}$ above those measured in a frequency range below $1 \mathrm{kHz}$. Another reason for the differences at high frequencies in the present case may be the use of the CLEANSC beamforming algorithm. The CLEAN-SC was found in past trailing edge noise studies [21] to give good results at low frequencies, but to deliver sound pressure levels that are lower than results obtained by the DAMAS algorithm and the orthogonal beamforming algorithm at high frequencies. However, the agreement between the spectral slope of the measured leading edge noise and that predicted by Amiet's model is quite good.

Besides the prediction based on Amiet's model, both Figure 12(a) and Figure 12(b) contain the autospectrum of one of the inner array microphones, which is positioned approximately above the airfoil leading edge normal to the chord line. As would be expected, the result from the single microphone exceeds the beamforming results, since it contains not only the noise source at the leading edge, but all noise sources present. This includes primarily the noise sources at the turbulence grid and also the noise due to the wind tunnel shear layer interacting with the airfoil as well as airfoil trailing edge noise. The increase of the single microphone autospectrum at high frequencies cannot be attributed to airfoil leading edge noise and is hence assumed to originate from other noise sources.

The sound pressure level spectra shown in Figure 12(a) and Figure 12(b) confirm that the porous airfoils lead to a noticeable noise reduction compared to the nonporous reference airfoil. For the PPS 12/2 turbulence grid (Figure 12(a)), the leading edge noise generated by the airfoil made of M-Pore $\mathrm{Al} 45 \mathrm{ppi}\left(r=1,000 \mathrm{~Pa} \mathrm{~s} / \mathrm{m}^{2}\right)$ exceeds the noise from the reference airfoil at high frequencies. Apart from this exception, the results indicate that porous airfoils with low air flow resistivities enable a larger noise reduction than porous airfoils with a high air flow resistivity. This is assumed to be caused by the (on average) larger pores of the materials with low air flow resistivities.

\section{Conclusions}

The present paper describes the use of microphone array beamforming for the investigation of airfoil leading edge noise. As an example application for this measurement technique, the potential noise reduction that can be achieved 


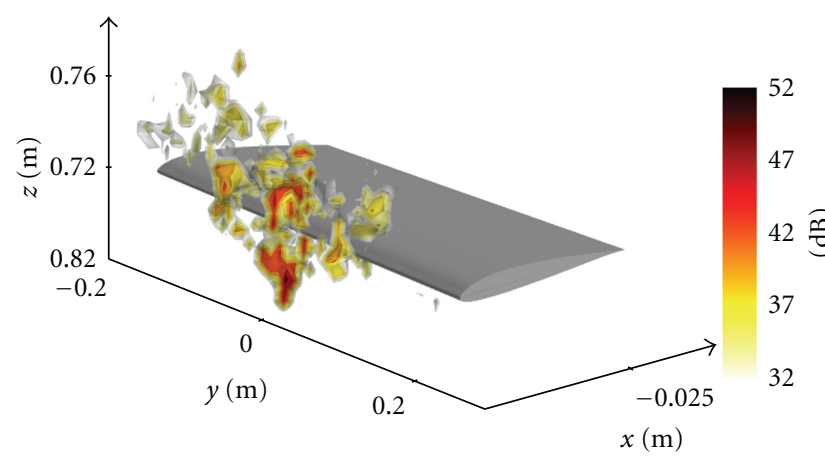

(a) Nonporous reference airfoil, $r=\infty$

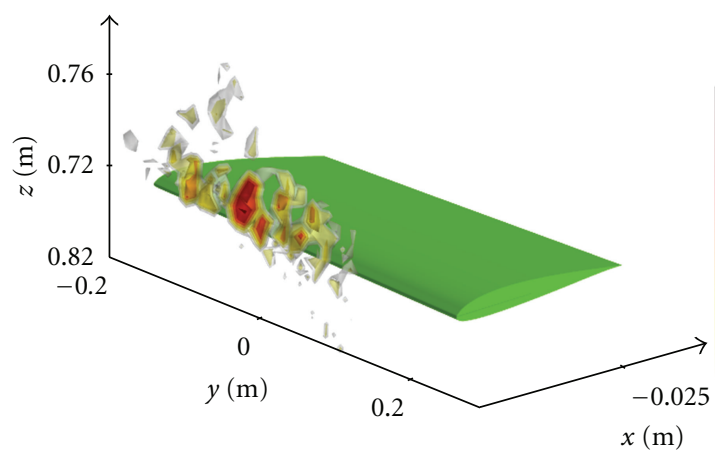

(c) Porous airfoil made of Recemat, $r=8,200 \mathrm{~Pa} / \mathrm{m}^{2}$

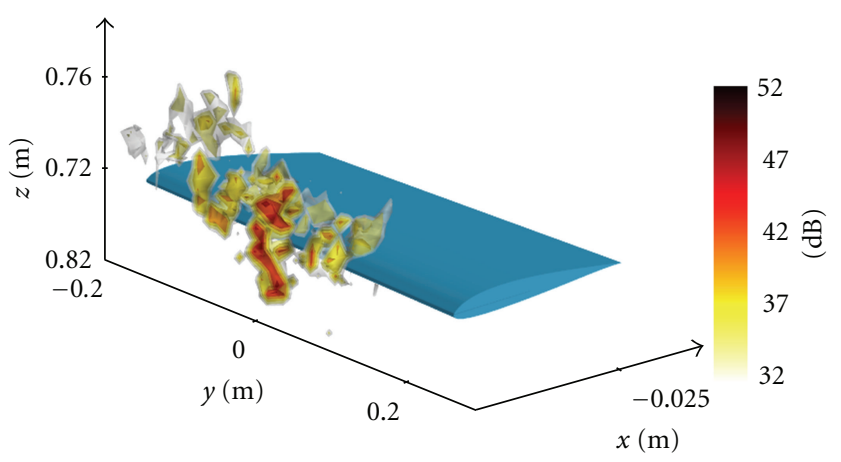

(b) Porous airfoil made of Needlona felt, $r=130,200 \mathrm{~Pa} \mathrm{~s} / \mathrm{m}^{2}$

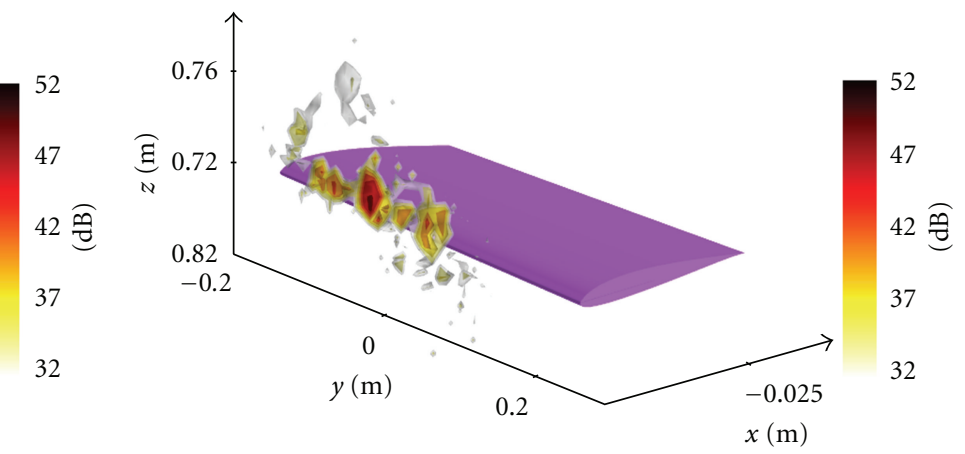

(d) Porous airfoil made of M-Pore Al $45 \mathrm{ppi}, r=1,000 \mathrm{~Pa} \mathrm{~s} / \mathrm{m}^{2}$

Figure 11: Three-dimensional third-octave band sound maps, PPS 14/4 turbulence grid, $U_{0}=35 \mathrm{~m} / \mathrm{s}(\mathrm{Re}=548,000)$, CLEAN-SC beamforming algorithm, center frequency $2 \mathrm{kHz}$. (Note that all sound maps are scaled to the same maximum level measured for the reference airfoil.).

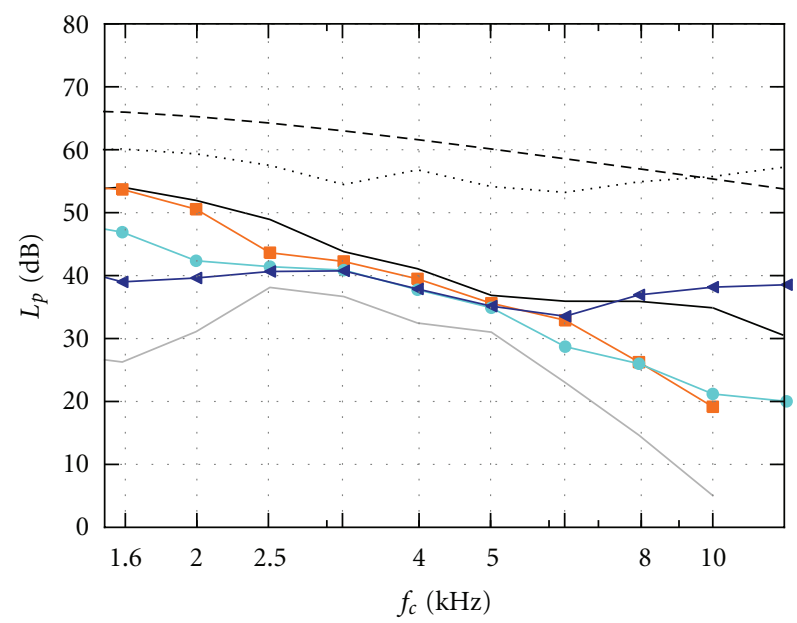

(a) PPS $12 / 2$ turbulence grid

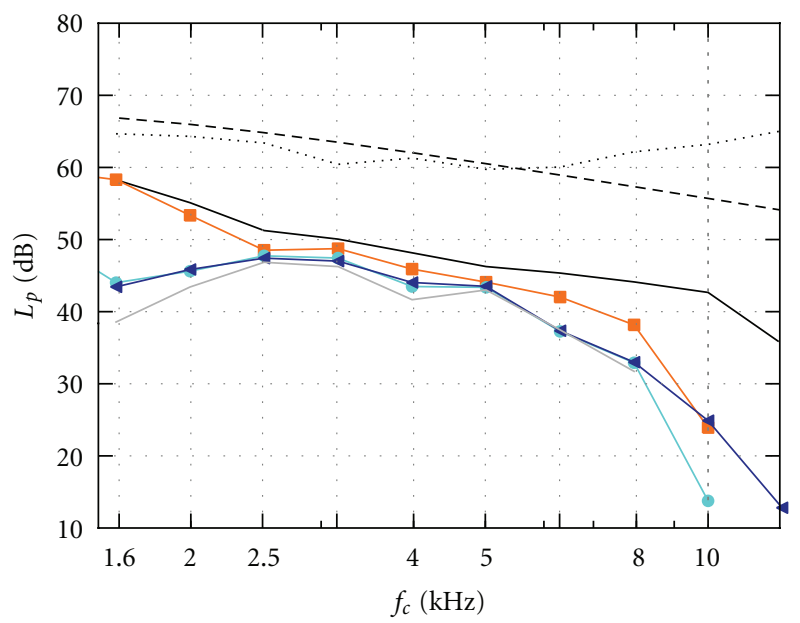

(b) PPS 14/4 turbulence grid

FIGURE 12: Leading edge noise third-octave band sound pressure levels, nominal flow speed $U_{0}=35 \mathrm{~m} / \mathrm{s}(\operatorname{Re}=548,000), r=-\infty,($ orange rectangle) 130,200, (faint blue circle) 8,200, (dark blue triangle) 1,000 $\mathrm{Pa} \mathrm{s} / \mathrm{m}^{2}$, (grey - ) empty test section, - - - Amiet model [5] according to $(1), \cdots$ auto-spectrum of one single array microphone for a measurement at the nonporous reference airfoil.

through the use of porous airfoils compared to a nonporous reference airfoil is investigated. To this end, detailed experiments were conducted in an open jet wind tunnel. Two turbulence grids provided the incident turbulence necessary for the generation of leading edge noise.
The acoustic measurements were performed with a planar 56 channel microphone array, positioned above the airfoils and out of flow. The acoustic data were processed using the CLEAN-SC algorithm, which was extended to account for a three-dimensional distribution of noise source 
locations. From the resultant three-dimensional sound maps, third-octave band sound pressure level spectra were obtained through integration over a volume that contains the leading edge noise sources at midspan but no other potential noise source locations, as for example the turbulence grid or the airfoil trailing edge.

Detailed hot-wire measurements with a single-wire probe were performed on several measurement locations in a plane normal to the flow, located upstream of the leading edge of the reference airfoil, in order to characterize the incident turbulence.

The leading edge noise spectra measured for the nonporous reference airfoil were compared to the predictions using the model developed by Amiet. The measured spectra are clearly below the predicted spectra. This is assumed to be due to the relatively low Mach number of the present experiments and, at higher frequencies, to the use of the CLEAN-SC beamforming. The results of the acoustic measurements show that the porous airfoils generate less leading edge noise than the nonporous reference airfoil.

\section{Acknowledgments}

This research was sponsored by the Deutsche Forschungsgemeinschaft (DFG) in the priority Program 1207, "Nature Inspired Fluid Mechanics," under the Grant no. SA 1502/1-3. The authors thank M. Hobracht for his help with the acoustic measurements.

\section{References}

[1] I. J. Sharland, "Sources of noise in axial flow fans," Journal of Sound and Vibration, vol. 1, no. 3, pp. 302-322, 1964.

[2] F. W. Grosveld, "Prediction of broadband noise from horizontal axis wind turbines," Journal of Propulsion and Power, vol. 1, no. 4, pp. 292-299, 1985.

[3] M. R. Fink, "Investigation of scrubbing and impingement noise,” Tech. Rep. NASA Contractor Report CR-134762, 1975.

[4] M. R. Fink, "Experimental evaluation of trailing edge and incidence uctuation noise theories," in the 13th AIAA Aerospace Sciences Meeting, 1975, AIAA- paper 75-206.

[5] R. K. Amiet, "Acoustic radiation from an airfoil in a turbulent stream," Journal of Sound and Vibration, vol. 41, no. 4, pp. 407420, 1975.

[6] R. W. Paterson and R. K. Amiet, "Acoustic radiation and surface pressure characteristics of an airfoil due to incident turbulence," Tech. Rep. NASA Contractor Report CR-2733, 1976.

[7] M. V. Lowson, "Assessment and prediction of wind turbine noise," Tech. Rep. Flow Solutions Ltd Contractor Report 92/19, 1992.

[8] S. Oerlemans, "Wind tunnel aeroacoustic tests of six airfoils for use on small wind turbines," Tech. Rep. NREL/SR-50035339, National Renewable Energy Laboratory, August 2004.

[9] S. Oerlemans and P. Migliore, "Aeroacoustic wind tunnel tests of wind turbine airfoils," Tech. Rep. NLR-TP-2004-319, National Aerospace Laboratory, 2004.

[10] S. Moreau and M. Roger, "Effect of angle of attack and airfoil shape on turbulence-interaction noise," in the 11th AIAA/CEAS Aeroacoustics Conference, vol. 3, pp. 2178-2198, March 2005.
[11] J. K. Staubs, Real Airfoil Effects on Leading Edge Noise, Ph.D. thesis, Virginia Polytechnic Institute and State University, 2008.

[12] F. V. Hutcheson, T. F. Brooks, C. L. Burley, and D. J. Stead, "Measurement of the noise resulting from the interaction of turbulence with a lifting surface," in the 17th AIAA/CEAS Aeroacoustics Conference, 2011, AIAA-paper 2011-2907.

[13] P. Sijtsma, "Clean based on spatial source coherence," in the 13th AIAA/CEAS Aeroacoustics Conference, 2007, AIAA-paper 2007-3436.

[14] E. Sarradj, C. Fritzsche, T. Geyer, and J. Giesler, "Acoustic and aerodynamic design and characterization of a small-scale aeroacoustic wind tunnel," Applied Acoustics, vol. 70, no. 8, pp. 1073-1080, 2009.

[15] J. O. Hinze, Turbulence, McGraw-Hill, New York, NY, USA, 2nd edition, 1975.

[16] J. S. Bendat and A. G. Piersol, Random Data-Analysis and Measurement Procedures, John Wiley \& Sons, New York, NY, USA, 3rd edition, 2000.

[17] G. G. Katul and M. B. Parlange, "Analysis of land surface heat fluxes using the orthonormal wavelet approach," Water Resources Research, vol. 31, no. 11, pp. 2743-2749, 1995.

[18] P. L. O’Neill, D. Nicolaides, D. Honnery, and J. Soria, "Autocorrelation functions and the determination of integral length with reference to experimental and numerical data," in the 15th Australasian Fluid Mechanics Conference, 2004.

[19] P. E. Roach, "The generation of nearly isotropic turbulence by means of grids," International Journal of Heat and Fluid Flow, vol. 8, no. 2, pp. 82-92, 1987.

[20] O. Ertunc, Experimental and Numerical Investigations of Axisymmetric Turbulence, Ph.D. thesis, Technical University Erlangen-Nürnberg, 2007.

[21] T. Geyer, E. Sarradj, and C. Fritzsche, "Measurement of the noise generation at the trailing edge of porous airfoils," Experiments in Fluids, vol. 48, no. 2, pp. 291-308, 2010.

[22] T. Geyer, E. Sarradj, and C. Fritzsche, "Porous airfoils: noise reduction and boundary layer effects," International Journal of Aeroacoustics, vol. 9, no. 6, pp. 787-820, 2010.

[23] ISO 9053, "Acoustics - materials for acoustical applications determination of airow resistance," Tech. Rep., International Organization for Standardization, 1993.

[24] R. Dougherty, "Beamforming in acoustic testing," in Aeroacoustic Measurements, pp. 62-97, Springer, Berlin, Germany, 2002.

[25] T. Suzuki, "A review of diagnostic studies on jet-noise sources and generation mechanisms of subsonically convecting jets," Fluid Dynamics Research, vol. 42, no. 1, Article ID 014001, 2010.

[26] S. Oerlemans, L. Broersma, and P. Sijtsma, "Quantification of airframe noise using microphone arrays in open and closed wind tunnels," International Journal of Aeroacoustics, vol. 6, no. 4, pp. 309-331, 2007.

[27] P. Sijtsma and R. W. Stoker, "Determination of absolute contributions of aircraft noise components using y-over array measurements," Tech. Rep. NLRTP-2004-167, National Aerospace Laboratory NLR, 2004.

[28] T. J. Mueller, Ed., Aeroacoustic Measurements, Springer, Berlin, Germany, 2002.

[29] E. Sarradj, "A fast signal subspace approach for the determination of absolute levels from phased microphone array measurements," Journal of Sound and Vibration, vol. 329, no. 9, pp. 1553-1569, 2010.

[30] T. F. Brooks and W. M. Humphreys, "A A deconvolution approach for the mapping of acoustic sources (DAMAS) 
determined from phased microphone arrays," in the 10th AIAA/CEAS Aeroacoustics Conference, pp. 1736-1753, May 2004.

[31] W. K. Blake, Mechanics of Flow-Induced Sound and Vibration, Volume II: Complex Flow-Structure Interactions, Academic Press, 1986.

[32] R. K. Amiet, "Refraction of sound by a shear layer," Journal of Sound and Vibration, vol. 58, no. 4, pp. 467-482, 1978.

[33] R. H. Schlinker and R. K. Amiet, "Refraction and scattering of sound by a shear layer,” Tech. Rep. NAS1-15339, NASA, Langley Research Center under Contract, 1980.

[34] J. Sanford, "Acoustic refraction: a three-dimensional shear layer correction for microphone measurements in an open-jet acoustic wind tunnel," Journal of Undergraduate Research, vol. 10, no. $1,2008$.

[35] P. T. Soderman and C. S. Allen, "Microphone measurements in and out of airstream," in Aeroacoustic Measurements, Springer Science/Business Media, Berlin, Germany, 2002.

[36] L. Sachs, Angewandte Statistik, Springer, Berlin, Germany, 1984.

[37] J. Gershfeld, "Leading edge noise from thick foils in turbulent flows," Journal of the Acoustical Society of America, vol. 116, no. 3, pp. 1416-1426, 2004. 

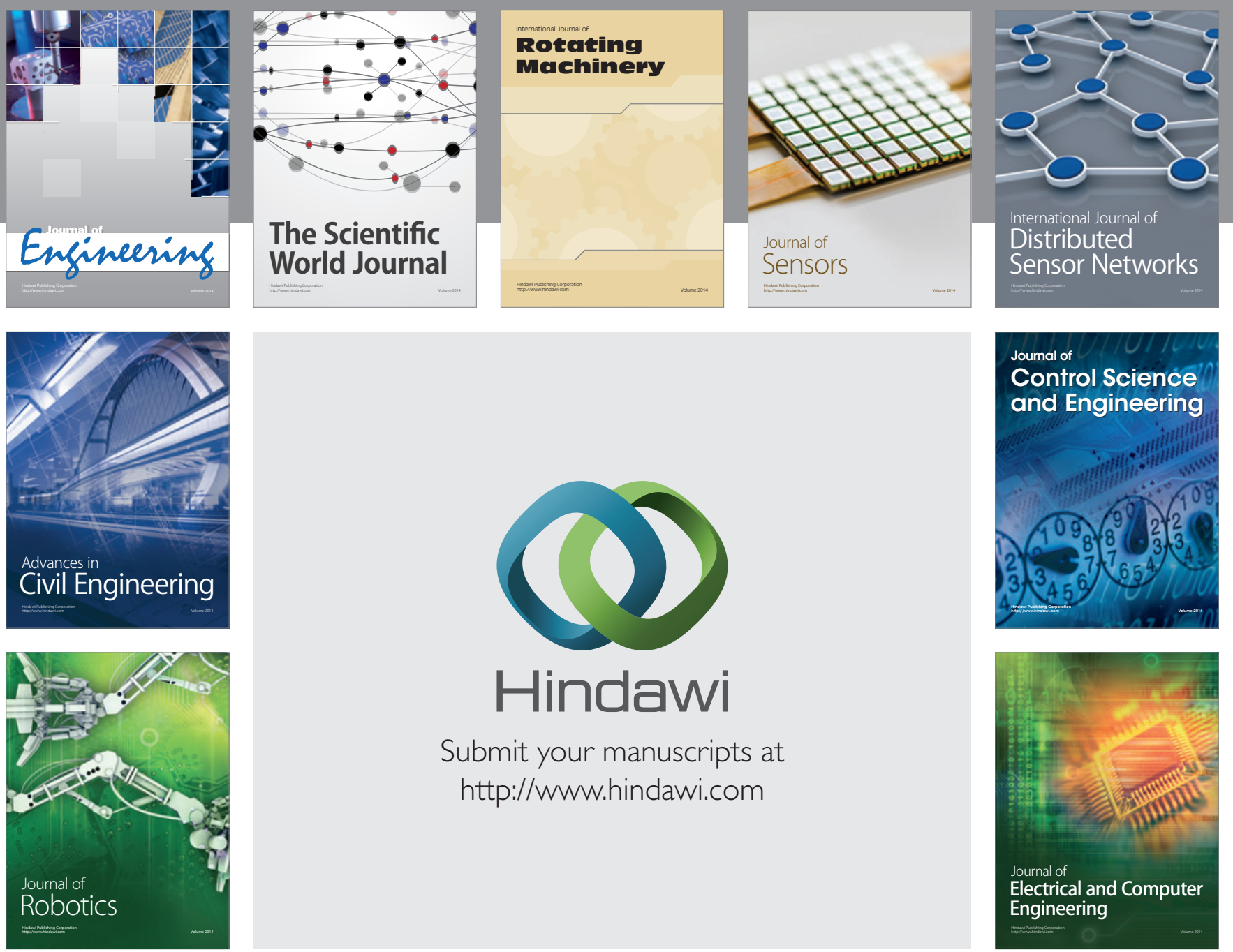

Submit your manuscripts at

http://www.hindawi.com
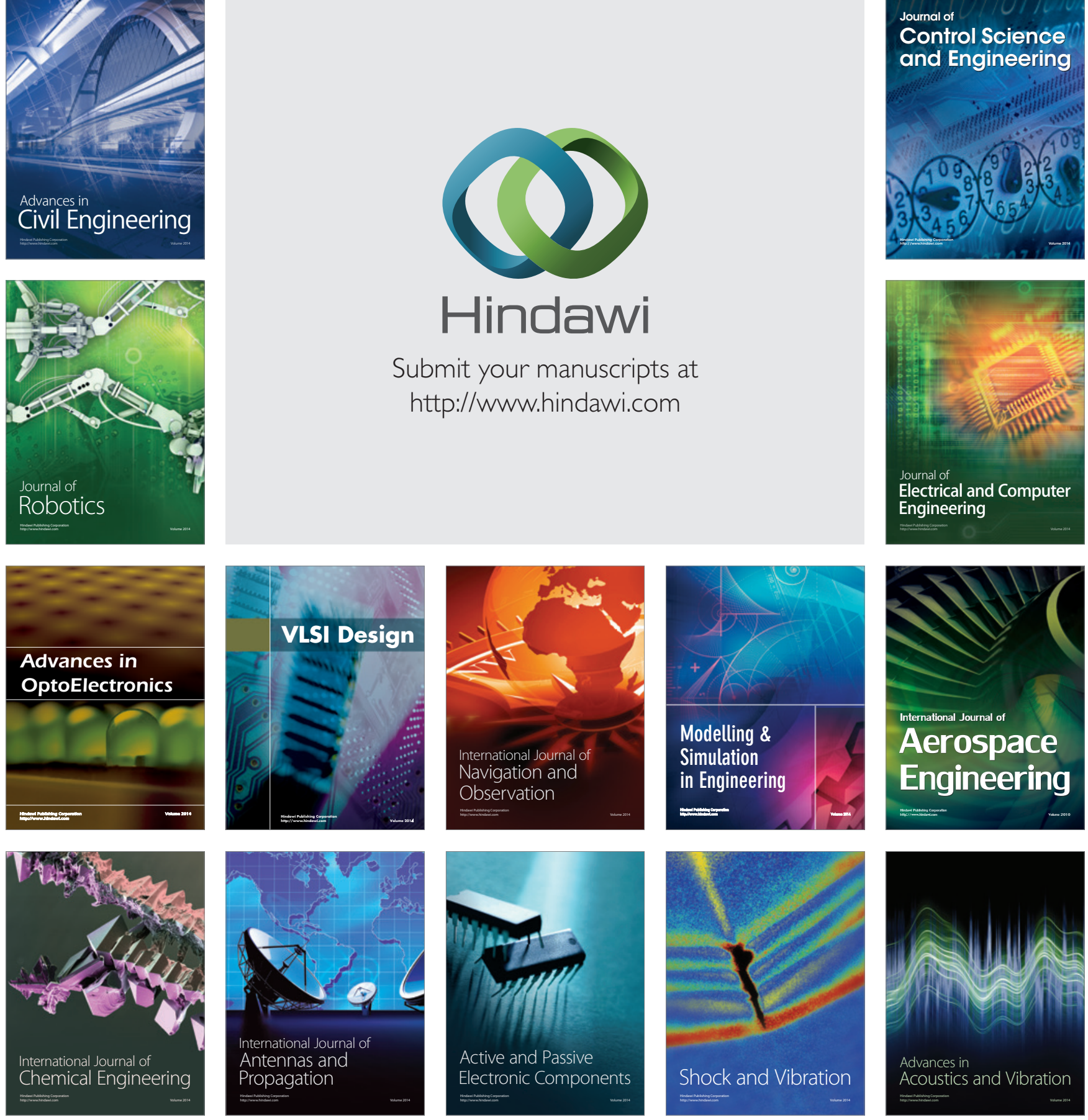